\title{
Direct Adaptive Control of Systems with Actuator Failures: State of the Art and Continuing Challenges
}

\author{
Gang Tao* $\quad$ Suresh M. Joshi ${ }^{\dagger}$
}

\begin{abstract}
In this paper, the problem of controlling systems with failures and faults is introduced, and an overview of recent work on direct adaptive control for compensation of uncertain actuator failures is presented. Actuator failures may be characterized by some unknown system inputs being stuck at some unknown (fixed or varying) values at unknown time instants, that cannot be influenced by the control signals. The key task of adaptive compensation is to design the control signals in such a manner that the remaining actuators can automatically and seamlessly take over for the failed ones, and achieve desired stability and asymptotic tracking. A certain degree of redundancy is necessary to accomplish failure compensation. The objective of adaptive control design is to effectively use the available actuation redundancy to handle failures without the knowledge of the failure patterns, parameters, and time of occurrence. This is a challenging problem because failures introduce large uncertainties in the dynamic structure of the system, in addition to parametric uncertainties and unknown disturbances. The paper addresses some theoretical issues in adaptive actuator failure compensation: actuator failure modeling, redundant actuation requirements, plant-model matching, error system dynamics, adaptation laws, and stability, tracking, and performance analysis. Adaptive control designs can be shown to effectively handle uncertain actuator failures without explicit failure detection. Some open technical challenges and research problems in this important research area are discussed.
\end{abstract}

\section{Introduction}

Actuator failures can cause control system performance deterioration and even lead to instability and catastrophic accidents (for example, United Flight 232, Sioux City, 1989: hydraulics/multiple actuator failures; USAir Flight 427, Pittsburgh, 1994: rudder failure; Alaska Flight 261, Pt. Mugu, 2000: stabilizer failure). Most of the safety problems posed by such failures still remain unsolved from the control theoretic as well as implementation points of view, because of the large structural uncertainties introduced by failures. Therefore it is important to investigate flight control system design techniques for enhanced survivability and reliability, with autonomous retention of stability and maneuverability when abnormal conditions occur.

Adaptive control methodology provides adaptation mechanisms that adjust a controller for a system subjected to parametric, structural, and environmental uncertainties to achieve both stability and tracking performance. Flight conditions, payload variation, and component aging cause parametric uncertainties, while component failures and damage lead to structural uncertainties. External disturbances (such as weather conditions and wind gusts) represent typical environmental uncertainties. The field of adaptive control has seen many important advances and remarkable successes in both theory and applications and continues to develop rapidly with the emergence of new challenging problems and their encouraging solutions. The ability to handle large, uncertain, and rapid structural as well as parametric changes caused by system failures or damage, is critical for resilient aircraft flight control.

Failures and damage in aircraft can be uncertain in that it is not known when, how many components have failed, which components have failed, the (quantitative) extent of the failures; or what type of damage has occurred, its magnitude, as well as its effect on the system dynamics. Compared to other approaches, the direct adaptive control approach has the key advantage that it can provide theoretically provable asymptotic

\footnotetext{
*Professor, Department of Electrical and Computer Engineering, University of Virginia, Charlottesville, VA, 22904; Email gt9s@virginia.edu

${ }^{\dagger}$ Senior Scientist, Mail Stop 308, NASA Langley Research Center, Hampton, VA 23681; Email suresh.m.joshi@nasa.gov; Fellow-AIAA.
} 
tracking in addition to stability, in the presence of large parameter variation and uncertainties. Furthermore, direct adaptive control designs do not require explicit failure detection and can directly adapt to failure uncertainties. Direct adaptation of feedback controller parameters, based on the difference between the desired and actual response, can be designed to be faster and more efficient in handling parametric and structural uncertainties caused by aircraft failures and damage. Direct adaptive control systems can make effective use of the available actuation redundancy for failure compensation. When failures or damage occur, desired stability and tracking performance (maneuverability) can still be achieved with adaptive cooperation of the remaining (non-failed) actuators to provide failure compensation and dynamic control.

Several approaches have been proposed in the literature for control of systems with failures. One approach to adaptive control consists of multiple model, switching and tuning schemes ${ }^{1-3}$. Another class of adaptive control designs is based on direct adaptive control approach $\left({ }^{4-6}\right.$ and the authors' work $\left.^{7-11}\right)$ for unknown systems with unknown actuator failures characterized by inputs stuck at some fixed or varying values not influenced by control action. Fault detection and diagnosis methods ${ }^{12-21}$ have also been proposed. Reconfigurable flight control designs using neural networks ${ }^{2-26}$ have been developed for aircraft systems with failures. Results on control of systems with failures also include those using: fault tolerant control designs $^{27-30}$; identification of multiplicative faults based on parameter estimation techniques ${ }^{31-34}$; function approximations for control and adaptive law design ${ }^{35}$; residual generation techniques for fault detection and diagnosis ${ }^{21,36-41}$; and other design and analysis techniques. ${ }^{42-45}$ In addition, direct adaptive reconfigurable control of a tailless fighter aircraft was presented and successfully flight-tested, ${ }^{26}$ and an adaptive controller for failure compensation in uncertain systems was presented. ${ }^{46}$

Despite recent progress, there are still open issues and challenges in adaptive control of systems with large, abrupt, and uncertain dynamics changes and failures. One important open research problem is how to design adaptive failure compensation control schemes that can analytically and provably ensure stability and asymptotic tracking properties in the presence of uncertain failures, for multivariable and nonlinear dynamic systems.

The purpose of this paper is to present a unified summary of the authors' recent work on developing direct adaptive control techniques for compensation of uncertain actuator failures and address some key technical issues: actuator failure modeling, redundant actuation requirement, plant-model matching, error system dynamics, adaptation laws, as well as stability, tracking, and performance analysis. Some open technical challenges and research problems are also discussed. The focus of the paper is on presenting theoretical results including the conceptual formulation, mathematical framework, and adaptive laws. Section II presents a formulation of the basic problems of direct adaptive control for systems with uncertain actuator failures. In Section III, a design method and related issues in adaptive compensation of actuator failures using state feedback for state tracking (SFST) are presented. Section IV presents adaptive compensation of actuator failures using state feedback for output tracking (SFOT). In Section V, adaptive compensation of actuator failures using output feedback for output tracking (OFOT) is addressed. Although the focus of the paper is mainly on linear systems, brief remarks and references regarding extensions to nonlinear systems are included.

\section{Basic Problem Formulation}

To formulate the actuator failure compensation problem, consider a dynamic system (plant)

$$
\dot{x}(t)=f(x(t), u(t), t), y(t)=h(x(t), t),
$$

where $x(t) \in R^{n}, y(t) \in R^{M}$, are the state and output vectors and $u(t)=\left[u_{1}, \ldots, u_{m}\right]^{T} \in R^{m}$ is the input vector whose components (actuators) may fail during system operation. One type of practical actuator failure is modeled as

$$
u_{j}(t)=\bar{u}_{j}, t \geq t_{j}, j \in\left\{j_{1}, j_{2}, \ldots, j_{p}\right\} \subset\{1,2, \ldots, m\}
$$

where the failure pattern $\left\{j_{1}, j_{2}, \ldots, j_{p}\right\}$, the failure value $\bar{u}_{j}$, and the failure time of occurrence $t_{j}$ are all unknown. For example, an aircraft control surface (such as the rudder or an aileron) may be locked at some unknown fixed value due to hydraulics failure. A more general failure model ${ }^{11}$ can be represented as

$$
u_{j}(t)=\bar{u}_{j}+\sum_{k=1}^{l_{j}} \bar{d}_{j k} \omega_{j k}(t)+\delta_{j}(t), t \geq t_{j}, j \in\left\{j_{1}, j_{2}, \ldots, j_{p}\right\},
$$


for some unknown constants $\bar{u}_{j}, \bar{d}_{j k} \in R$ and bounded disturbance $\delta_{j}(t) \in R$, and known signals $\omega_{j k}(t) \in R$ (which may also depend on the system state $\left.x(t): \omega_{j k}(t)=\omega_{j k}(x(t), t)\right), j=1,2, \ldots, m, k=1,2, \ldots, l_{j}$. This failure model can approximate a large class of practically meaningful failures in control applications.

Let $v(t)=\left[v_{1}, v_{2}, \ldots, v_{m}\right]^{T} \in R^{m}$ be the applied (commanded) control input vector. In the presence of actuator failures as in (2) or (3), the actual input vector $u$ to the system can be described as

$$
u(t)=v(t)+\sigma(\bar{u}-v(t)),
$$

where $\bar{u}=\left[\bar{u}_{1}, \bar{u}_{2}, \ldots, \bar{u}_{m}\right]^{T}, \sigma=\operatorname{diag}\left\{\sigma_{1}, \sigma_{2}, \ldots, \sigma_{m}\right\}$, with $\sigma_{i}=1$ if the $i$ th actuator fails, i.e., $u_{i}=\bar{u}_{i}$, or $\sigma_{i}=0$ otherwise. That is, $\sigma$ is a diagonal matrix whose entries are piecewise step- or zero functions of time. Some components of the applied input signal $v(t)=\left[v_{1}(t), v_{2}(t), \ldots, v_{m}(t)\right]^{T}$, that is, those $v_{i}(t)$ with $\sigma_{i}=1$, cannot affect the system dynamics. The actuator failures are uncertain in value, pattern and time of occurrence.

The control signal $v(t)$ should be synthesized so as to ensure the system stability and asymptotic tracking performance regardless of whether (or which) actuators have failed, or the failure values. That is, $v(t)$ should be capable of compensating for the failures automatically.

The actuator failures are assumed to occur instantaneously, i.e., $\sigma_{i}$ are piecewise constant functions of time. Since there are $2^{m}$ possible combinations of actuator states (each actuator is either normal or failed), there are $2^{m}-1$ possible forms of failure patterns, and at any point in time, the failure pattern is represented by the matrix $\sigma=\operatorname{diag}\left\{\sigma_{1}, \sigma_{2}, \ldots, \sigma_{m}\right\}$ (including the no-failure case but excluding the all-failure case). However, for a specific application, not all failure patterns are expected to occur, and it is of interest to consider the compensation problem for failures whose patterns belong to a non-empty subset $\Sigma$ of $m \times m$ diagonal matrices with "0" or "1" as entries, that is, a desired controller should be able to accommodate all failure patterns within $\Sigma$ (which also contains the no-failure case). For some applications, it may be of interest to consider the failure compensation problem for up to some $q(1 \leq q \leq m-1)$ actuator failures. In this case, there are totally $\sum_{i=0}^{q}\left(\begin{array}{c}m \\ i\end{array}\right)$ possible failure patterns, including the no-failure case.

The control objective is to design adaptive feedback control schemes to achieve system stability and asymptotic state or output tracking in the presence of actuator failures whose patterns $\sigma$ belong to a failure pattern set $\Sigma$. The key task of adaptive actuator failure compensation control is to adaptively adjust the remaining controls (unfailed actuators) to achieve the desired system performance, without the knowledge of the system and failure parameters.

The relevant adaptive control problems can be classified into three groups:

(i) state feedback design for state tracking (SFST),

(ii) state feedback design for output tracking (SFOT), and

(iii) output feedback design for output tracking (OFOT).

State feedback designs use simpler controller structures as compared with output feedback designs, while state tracking needs stronger conditions on the system as compared with output tracking. Clarification of such design conditions is important for specifying guidelines for designing control systems for adaptive failure compensation.

Redundancy. Component redundancy has been widely employed in the design of modern control systems such as aircraft flight control systems, flexible space structures, and other performance-critical systems. Redundancy is necessary for failure compensation, and brings new challenges for control design, especially, when the failure pattern is unknown. For example, a nominal system may have 5 actuators and up to 4 of them may fail during system operation. Without knowing which actuators have failed, control input signals have to be designed for all 5 actuators such that the remaining actuators can still control the system. An ideal adaptive controller should be able to handle all possible failure patterns and values.

Failure uncertainties. Component failures introduce large system uncertainties. For example, when some actuators fail, the system structure from the active inputs (unfailed actuators) to the output experiences significant changes, as do the system parameters. Furthermore, failures cause additional disturbances in the system which influence the system's behavior. Compensation of such disturbances can be a challenging problem (i.e., certain matching conditions have to be established). In this sense, failures do not merely cause system parameter or gain changes, but they lead to system uncertainties that cannot be treated by standard 
adaptive control designs. In addition to stabilization (signal boundedness), asymptotic tracking/regulation is also needed in the presence of failures, particularly in aircraft flight control applications.

The technical goal is to achieve both closed-loop stabilization and asymptotic tracking for systems in the presence of uncertain structural and parametric uncertainties caused by failures and damage with unknown values and patterns, in addition to other system and dynamics uncertainties. As stated previously, three types of adaptive control problems for failure compensation with increasing complexity are considered, i.e., SFST, SFOT, and OFOT. Recent results are presented in a unified framework, and key challenges in this research are discussed. The research is initially focused on linear time-invariant systems (plants) described by

$$
\dot{x}(t)=A x(t)+B u(t), y(t)=C x(t),
$$

where $A \in R^{n \times n}, B \in R^{n \times m}, C \in R^{M \times n}$ with $M<m, x(t) \in R^{n}, y(t) \in R^{M}$, and $u(t) \in R^{m}$ whose components may fail as described by (2) or (3). Some extensions of the results to nonlinear system of the form (1) are also briefly discussed.

\section{Adaptive State Feedback Design for State Tracking}

For the state feedback state tracking (SFST) problem, we consider the plant

$$
\dot{x}(t)=A x(t)+B u(t), x(t) \in R^{n}, u(t) \in R^{m},
$$

The control objective is to design an adaptive state feedback control signal $v(t) \in R^{m}$ to be applied to all $m$ actuators so that the closed-loop signal boundedness and asymptotic tracking are ensured despite uncertain actuator failures, i.e., $\lim _{t \rightarrow \infty}\left(x(t)-x_{m}(t)\right)=0$, where $x_{m}(t)$ is a given desired reference state trajectory.

In the presence of actuator failures, in view of (4), the system (6) can be rewritten as

$$
\dot{x}(t)=A x(t)+B\left(I_{m}-\sigma\right) v(t)+B \sigma \bar{u},
$$

where $I_{m}$ is the $m \times m$ identity matrix. The objective is to synthesize the signal $v(t)$ which achieves the control objectives despite unknown failures $\sigma \in \Sigma$ and accompanying disturbances $\bar{u}$.

\section{III.A. Adaptive Control Design}

The plant parameters $A$ and $B$ are usually not known accurately. The objective of SFST adaptive control is to track the state trajectory of a reference model that embodies the desired closed-loop behavior. The choice of the reference model system for $x_{m}(t)$ depends on certain structure conditions on $A$ and $B$, and so does the design of the parameter adaptation laws. The reference model system is represented by

$$
\dot{x}_{m}(t)=A_{m} x_{m}(t)+B_{m} r(t),
$$

where $r(t) \in R^{m}$ is a bounded reference input signal, $A_{m} \in R^{n \times n}$ is stable, and $B_{m} \in R^{n \times m}$, and the following assumptions are made:

(A.1): $(A, B)$ is controllable, and $\operatorname{rank}\left[B\left(I_{m}-\sigma\right)\right]=\operatorname{rank}[B], \forall \sigma \in \Sigma$ at any point in time.

(A.2): $A_{m}=A+B K$ for some $m \times n$ gain matrix $K$; and $B\left(I_{m}-\sigma\right) \kappa_{\sigma}=B_{m}$ for some nonsingular gain matrix $\kappa_{\sigma}=\kappa(\sigma) \in R^{m \times m}$, for each $\sigma \in \Sigma$, at any point in time.

Note that only the existence of such $K$ and $\kappa_{\sigma}$ is assumed, and their values are not needed. The rank condition in Assumption (A.1) implies that adequate actuator redundancy exists, and Assumption (A.2) implies that some structural conditions on $A, B, A_{m}$ and $B_{m}$ are needed.

Consider the adaptive control law

$$
v(t)=\widehat{K}(t) x(t)+\widehat{\kappa}(t) r(t)+\widehat{\theta}(t),
$$

where $\widehat{K}=\left[\widehat{K}_{1}, \widehat{K}_{2}, \ldots, \widehat{K}_{m}\right]^{T} \in R^{m \times n}, \widehat{\kappa}=\left[\widehat{\kappa}_{1}, \widehat{\kappa}_{2}, \ldots, \widehat{\kappa}_{m}\right]^{T} \in R^{m \times m}$, and $\widehat{\theta}=\left[\widehat{\theta}_{1}, \widehat{\theta}_{2}, \ldots, \widehat{\theta}_{m}\right]^{T} \in R^{m}$, are the estimates of the unknown parameters $K_{\sigma} \in R^{m \times n}, \kappa_{\sigma} \in R^{m \times m}$ and $\theta_{\sigma} \in R^{m}$, which exist because of assumptions (A.1) and (A.2), and satisfy

$$
B\left(I_{m}-\sigma\right) K_{\sigma}=B K, B\left(I_{m}-\sigma\right) \kappa_{\sigma}=B_{m}, B\left(I_{m}-\sigma\right) \theta_{\sigma}=-B \sigma \bar{u} .
$$


With this controller structure, for an actuator failure pattern $\sigma \in \Sigma$, the error equation can be derived as

$$
\begin{aligned}
\dot{e}(t) & =A_{m} e(t)+B\left(I_{m}-\sigma\right)\left(\left(\widehat{K}(t)-K_{\sigma}\right) x(t)+\left(\widehat{\kappa}(t)-\kappa_{\sigma}\right) r(t)+\left(\widehat{\theta}(t)-\theta_{\sigma}\right)\right) \\
& =A_{m} e(t)+B_{m} \kappa_{\sigma}^{-1}\left(\left(\widehat{K}(t)-K_{\sigma}\right) x(t)+\left(\widehat{\kappa}(t)-\kappa_{\sigma}\right) r(t)+\left(\widehat{\theta}(t)-\theta_{\sigma}\right)\right) .
\end{aligned}
$$

where $e(t)=x(t)-x_{m}(t)$.

This type of error model is familiar in the literature, ${ }^{47}$ and can be used to develop an adaptive scheme for updating the parameter estimates $\widehat{K}(t), \widehat{\kappa}(t)$ and $\widehat{\theta}(t)$ in the feedback controller (9), under the condition that there is a known $m \times m$ matrix $S$ such that $\kappa_{\sigma} S=\left(\kappa_{\sigma} S\right)^{T}>0$ for each $\sigma \in \Sigma$ (see pp. 372-374 of Ref. [47]) - such a condition is a standard condition for multivariable SFST model reference adaptive control (MRAC), even in the absence of actuator failures. The adaptation laws are given by

$$
\begin{aligned}
\dot{\hat{K}}(t) & =-S^{T} B_{m}^{T} P e(t) x^{T}(t) \\
\dot{\widehat{\kappa}}(t) & =-S^{T} B_{m}^{T} P e(t) r^{T}(t) \\
\dot{\hat{\theta}}(t) & =-S^{T} B_{m}^{T} P e(t)
\end{aligned}
$$

where $P=P^{T}>0$ is the solution of the Lyapunov equation: $A_{m}^{T} P+P A_{m}=-Q$ for a chosen matrix $Q=Q^{T}>0$.

\section{III.A.1. Stability Analysis}

This adaptive control scheme has the following desired properties:

Theorem 1 [8] The control law (9), updated from (12)-(14) and applied to the system (6) subject to the actuator failures (2), ensures that all closed-loop system signals are bounded and $\lim _{t \rightarrow \infty}\left(x(t)-x_{m}(t)\right)=0$.

The proof can be obtained by using a piecewise continuous Lyapunov function given by

$$
V=e^{T} P e+\operatorname{tr}\left[\widetilde{K}^{T} M_{\sigma}^{-1} \widetilde{K}\right]+\operatorname{tr}\left[\widetilde{\kappa}^{T} M_{\sigma}^{-1} \widetilde{\kappa}\right]+\operatorname{tr}\left[\widetilde{\theta}^{T} M_{\sigma}^{-1} \widetilde{\theta}\right],
$$

where $\operatorname{tr}[\cdot]$ is the trace of a matrix, $M_{\sigma}=\kappa_{\sigma} S, \widetilde{K}(t)=\widehat{K}(t)-K_{\sigma}, \widetilde{\kappa}(t)=\widehat{\kappa}(t)-\kappa_{\sigma}$ and $\widetilde{\theta}(t)=\widehat{\theta}(t)-\theta_{\sigma}$, with $K_{\sigma}, \kappa_{\sigma}, \theta_{\sigma}$ and $M_{\sigma}$ being piecewise constant (and unknown) parameters, so that $V$ is a piecewise continuous function. It can be verified that $\dot{V}=-e^{T}(t) Q e(t)$ for $t \in\left(T_{k}, T_{k+1}\right)$, from which it can be shown that all closed-loop system signals are bounded and $\lim _{t \rightarrow \infty}\left(x(t)-x_{m}(t)\right)=0$.

\section{III.B. Discussion}

There are several key technical issues in direct adaptive control of systems with uncertain actuator failures such as failure uncertainties, design conditions and stable adaptation, which are discussed next.

Failure uncertainties. Actuator failures cause system uncertainties even if the system parameters $A$ and $B$ were known. Such uncertainties are characterized by the uncertain parameters $K_{\sigma} \in R^{m \times n}, \kappa_{\sigma} \in R^{m \times m}$ and $\theta_{\sigma} \in R^{m}$ defined in the above subsections. The variations of such parameters can be large, and the adaptive control scheme must be designed to accommodate them. Actuator failure uncertainties and system parameter uncertainties together pose a challenging control problem, requiring rather restrictive design conditions for state tracking. In the presence of uncertain actuator failures, the design conditions include: one for failure compensation and one for plant-model matching, leading to those given in Assumptions (A.1) and (A.2); especially, the plant-model structural matching conditions for each failure pattern $\sigma \in \Sigma$.

- The condition that $B\left(I_{m}-\sigma\right) \kappa_{\sigma}=B_{m}$ for some nonsingular gain matrix $\kappa_{\sigma}=\kappa(\sigma)$ for each $\sigma \in \Sigma$, imposes certain constraints on the plant actuation matrix $B$ and the reference model system actuation matrix $B_{m}$. Such constraint can be necessary for certain applications. One such case is when an adaptive control scheme has to be able to effectively compensate for all $\sum_{i=0}^{q}\left(\begin{array}{c}m \\ i\end{array}\right)$ possible failure patterns corresponding to up to $q=m-1$ failures out of $m$ actuators. In Ref. [11], it was shown that 
the design conditions require the columns $b_{i}$ of $B=\left[b_{1}, b_{2}, \ldots, b_{m}\right]$ to be parallel to each other and also to the columns of $B_{m}$. Under this condition, it can be verified that $B\left(I_{m}-\sigma\right) \kappa_{\sigma}=B_{m}$ is satisfied for some nonsingular gain matrix $\kappa_{\sigma}$ for each $\sigma \in \Sigma$, and that the desired $S$ matrix for the adaptive laws (22)-(24) can be chosen to be diagonal.

As an example, consider the case of $m=3$. The design conditions for up to 2 failures of the total $m=3$ actuators require that $B=\left[b_{1}, b_{2}, b_{3}\right]=\left[a_{1} b, a_{2} b, a_{3} b\right]$ for some non-zero vector $b \in R^{n}$ and some scalars $a_{i} \neq 0, i=1,2,3$, and $B_{m}=\left[k_{1} b_{1}, k_{2} b_{2}, k_{3} b_{3}\right]$ for some scalars $k_{i} \neq 0, i=1,2,3$. For the no-failure pattern, that is, $\sigma=0$, we have $\kappa_{\sigma}=\operatorname{diag}\left\{k_{1}, k_{2}, k_{3}\right\}$, so that $B \kappa_{\sigma}=B_{m}$. The desired gain matrix $S$ for $\kappa_{\sigma} S=\left(\kappa_{\sigma} S\right)^{T}>0$ is $S=\operatorname{diag}\left\{s_{1}, s_{2}, s_{3}\right\}=\operatorname{diag}\left\{\operatorname{sign}\left[k_{1}\right] \gamma_{1}, \operatorname{sign}\left[k_{2}\right] \gamma_{2}, \operatorname{sign}\left[k_{3}\right] \gamma_{3}\right\}$, for any $\gamma_{i}>0, i=1,2,3$. Then, for the failure of the first actuator $u_{1}$, we have $\sigma=\operatorname{diag}\{1,0,0\}$, and $\kappa_{\sigma}=\left[\begin{array}{ccc}\alpha & \frac{a_{1} k_{1} s_{1}}{a_{2} s_{2}} & 0 \\ \frac{a_{1} k_{1}}{a_{2}} & k_{2} & 0 \\ 0 & 0 & k_{3}\end{array}\right]$, which lead to $B\left(I_{m}-\sigma\right) \kappa_{\sigma}=B_{m}$ and $\kappa_{\sigma} S=\left(\kappa_{\sigma} S\right)^{T}$, where $\alpha$ is such that $\alpha s_{1}>0$ and $\alpha s_{1} s_{2} k_{2}>\frac{s_{1}^{2} a_{1}^{2} k_{1}^{2}}{a_{2}^{2}}$ so that $\kappa_{\sigma} S>0$. For the failures of $u_{1}$ and $u_{2}$, we have $\sigma=\operatorname{diag}\{1,1,0\}$, and $\kappa_{\sigma}=\left[\begin{array}{ccc}\alpha_{1} & 0 & \frac{a_{1} k_{1} s_{1}}{a_{3} s_{3}} \\ 0 & \alpha_{2} & \frac{s_{2} a_{2} k_{2}}{a_{3} s_{3}} \\ \frac{a_{1} k_{1}}{a_{3}} & \frac{a_{2} k_{2}}{a_{3}} & k_{3}\end{array}\right]$, which lead to $B\left(I_{m}-\sigma\right) \kappa_{\sigma}=B_{m}$ and $\kappa_{\sigma} S=\left(\kappa_{\sigma} S\right)^{T}$ for the chosen $S$, where $\alpha_{1}$ and $\alpha_{2}$ are such that $\alpha_{1} s_{1}>0, \alpha_{2} s_{2}>0$, and $\operatorname{det}\left(\kappa_{\sigma} S\right)>0$ so that $\kappa_{\sigma} S>0$. The existence of the desired $\kappa_{\sigma}$ for each $\sigma \in \Sigma$ (which also contains $\operatorname{diag}\{0,1,0\}, \operatorname{diag}\{0,0,1\}, \operatorname{diag}\{0,1,1\}$, $\operatorname{diag}\{1,0,1\})$ can be similarly verified, for a fixed chosen $S=\operatorname{diag}\left\{\operatorname{sign}\left[k_{1}\right] \gamma_{1}, \operatorname{sign}\left[k_{2}\right] \gamma_{2}, \operatorname{sign}\left[k_{3}\right] \gamma_{3}\right\}$. (The signs of $k_{i}$ are usually known from the physics of the problem).

- The conditions that $A_{m}=A+B\left(I_{m}-\sigma\right) K_{\sigma}$ and $B \kappa_{\sigma}=B_{m}$ (for some $K_{\sigma}$ and $\kappa_{\sigma}$ ) in Assumption (A.2) are also needed for a standard (no failure- $\sigma=0$ ) SFST MRAC scheme. These conditions, suggest that the SFST adaptive control design has significant limitations for many applications, including aircraft flight control, in which the matching condition $A_{m}=A+B\left(I_{m}-\sigma\right) K_{\sigma}$ may be difficult to satisfy for realistic situations when $A$ and $B$ are unknown and may change over time. This is an open issue in the state tracking problem. However, it should be noted that, for systems that are in a canonical form (such as the Companion form), these conditions can always be satisfied if sufficient actuator redundancy exists.

- Another type of actuator failure uncertainty is reduced effectiveness, which can occur due to structural damage (e.g., loss of a part of a control surface or engine malfunction) or icing. This can be represented as:

$$
u=\Xi v
$$

where $\Xi$ is a diagonal matrix and $\Xi_{i, i} \in(0,1]$, which are unknown and uncertain. If reduced actuator effectiveness and actuator failures are simultaneously present, the system input is given by

$$
u=\Xi\left(I_{m}-\sigma\right) v+\sigma \bar{u}
$$

which results in

$$
\begin{aligned}
\dot{x} & =A x+B \Xi\left(I_{m}-\sigma\right) v+B \sigma \bar{u} \\
& =A x+B\left(I_{m}-\sigma\right) \Xi v+B \sigma \bar{u}
\end{aligned}
$$

Assuming that $\Xi$ is piecewise constant, all the analysis and results for the SFST case (with the control law given by Eq. (9)) carry over with the parameters $K_{\sigma}, \kappa_{\sigma}, \theta_{\sigma}$, (and their estimates) simply replaced by $\Xi K_{\sigma}, \Xi \kappa_{\sigma}$ etc.

Although $\Xi_{i, i} \in(0,1]$ represents reduced effectiveness, the analyses are also valid for $\Xi_{i, i} \in(0, \infty)$, which implies that the gain margin of the SFST adaptive control law is $(0, \infty)$.

Extension to nonlinear dynamic systems. Extension of the above results to nonlinear systems of the form: $\dot{x}(t)=f(x(t), u(t), t), y(t)=h(x(t), t)$, needs to address two main issues: (i) specification of a 
controller structure with complete parametrization of the system uncertainties caused by uncertain actuator failures, and (ii) development of an adaptive scheme for stable adaptation of the controller parameters. One feasible adaptive control scheme is based on the feedback linearization method, ${ }^{48}$ using SFST, and has been reported in Chapter 9 of Ref. [11]. This continues to be an area of further research.

Adaptive SFST design needs rather stringent matching conditions. However, it is usually not necessary to track the entire state vector, and it is sufficient to track only $M(<n)$ outputs. The next two sections address output tracking, using state feedback or output feedback.

\section{Adaptive State Feedback Design for Output Tracking}

For the state feedback output tracking (SFOT) adaptive control problem, consider the plant (5):

$$
\dot{x}(t)=A x(t)+B u(t), y(t)=C x(t)
$$

where $A \in R^{n \times n}, B=\left[b_{1}, b_{2}, \ldots, b_{m}\right] \in R^{n \times m}, C \in R^{M \times n}$ with $M<m, x(t) \in R^{n}, y(t) \in R^{M}$, and $u(t) \in R^{m}$. Some components of $u$ may fail as described by (2) or (3). The control objective is to design an adaptive state feedback control signal $v(t) \in R^{m}$ to be applied to all $m$ actuators subject to uncertain failures, to ensure closed-loop signal boundedness and asymptotic output tracking (i.e., $\lim _{t \rightarrow \infty}\left(y(t)-y_{m}(t)\right)=0$, where $y_{m}(t)$ is a given desired reference output trajectory) despite uncertain actuator failures.

The reference model system for generating $y_{m}(t)$ has the form

$$
\dot{x}_{m}(t)=A_{m} x_{m}(t)+B_{m} r(t), y_{m}(t)=C_{m} x_{m}(t)
$$

where $A_{m} \in R^{n_{r} \times n_{r}}$ is stable and known, $B_{m} \in R^{n_{r} \times m_{r}}$ and $C_{m} \in R^{M \times n_{r}}$ are both known, $x_{m}(t) \in R^{n_{r}}$ is the reference system state vector, and $r(t) \in R^{m_{r}}\left(1 \leq m_{r} \leq m\right)$ is a bounded reference input signal. The signal $x_{m}(t)$ is not explicitly relevant for control design because the tracking of the entire state vector is not the objective. This relaxes the restrictive matching conditions in Assumption (A.2). An input-output form reference model system is by

$$
y_{m}(t)=W_{m}(s)[r](t)
$$

for some stable $M \times m_{r}$ transfer matrix $W_{m}(s)$ to be specified.

For output tracking, the state feedback control structure is the same as (9):

$$
v(t)=\widehat{K}(t) x(t)+\widehat{\kappa}(t) r(t)+\widehat{\theta}(t),
$$

where $\widehat{K}=\left[\widehat{K}_{1}, \widehat{K}_{2}, \ldots, \widehat{K}_{m}\right]^{T} \in R^{m \times n}, \widehat{\kappa}=\left[\widehat{\kappa}_{1}, \widehat{\kappa}_{2}, \ldots, \widehat{\kappa}_{m}\right]^{T} \in R^{m \times m_{r}}$, and $\widehat{\theta}=\left[\widehat{\theta}_{1}, \widehat{\theta}_{2}, \ldots, \widehat{\theta}_{m}\right]^{T} \in R^{m}$ are parameters to be updated from some adaptive laws.

For effective compensation of actuator failures, it is important to obtain a complete parametrization of the system with parameter and failure uncertainties. For system parametrization, a nominal controller needs to be defined, that is, the parameters $K_{\sigma}, \kappa_{\sigma}$ and $\theta_{\sigma}$ required to achieve output tracking need to be characterized. These parameters are subsequently to be estimated by $\widehat{K}(t), \widehat{\kappa}(t)$ and $\widehat{\theta}(t)$. The desired output matching is expressed as: $y(t)=y_{m}(t)+\epsilon_{0}(t)$ for some exponentially decaying initial condition-related signal $\epsilon_{0}(t)$, and to achieve closed-loop signal boundedness for all $\sigma \in \Sigma$. In the SFOT case, system signal boundedness is related to internal stability, which depends on a certain minimum phase condition. Unlike the adaptive control problem without actuator failures wherein the plant (usually) remains minimum-phase despite parameter variations, some actuator failures may cause the plant zeros to migrate into the right-half plane. A desired controller parametrization should be able to ensure both system stability and plant-model output matching for any failure pattern $\sigma \in \Sigma$.

In this section, the plant-model matching issue is first addressed for the case where $M=1$ and $m_{r}=1$; that is, the reference model system is a single-input single-output system $y_{m}(t)=W_{m}(s)[r](t)$ with a scalar reference input $r(t)$ and a stable transfer function $W_{m}(s)$. (This notation is used to indicate a signal passed through a transfer function). Adaptive control schemes are then developed for the system $\dot{x}(t)=A x(t)+B u(t), y(t)=C x(t) \in R$ (for $M=1)$. The actuator failures are always assumed to be unknown.

The cases when $M>1$ (multi-output tracking) or/and $m_{r}>1$ (multiple reference inputs) are still under investigation. 


\section{IV.A. Plant-Model Matching}

To study the plant-model matching under failure conditions, consider the nominal controller

$$
v(t)=K x(t)+\kappa r(t)+\theta,
$$

where $K=\left[K_{1}, K_{2}, \ldots, K_{m}\right]^{T} \in R^{m \times n}, \kappa=\left[\kappa_{1}, \kappa_{2}, \ldots, \kappa_{m}\right]^{T} \in R^{m \times m_{r}}$, and $\theta=\left[\theta_{1}, \theta_{2}, \ldots, \theta_{m}\right]^{T} \in R^{m}$ are some nominal parameters. Recall that for a failure pattern $\sigma \in \Sigma$, we have $u=v+\sigma(\bar{u}-v)$. Suppose there are $p$ failed actuators associated with $\sigma$ at time $t$, that is, $u_{j}=\bar{u}_{j}, j=j_{1}, j_{2}, \ldots, j_{p}$, for some $j_{1}, j_{2}, \ldots, j_{p} \in\{1,2, \ldots, m\}$. Then the closed-loop system is given by

$$
\begin{aligned}
\dot{x}(t) & =(A+B K) x(t)+B \kappa r(t)+B \theta+B \sigma(\bar{u}-v(t)) \\
& =\left(A+\sum_{i \neq j_{1}, \ldots, j_{p}} b_{i} K_{i}^{T}\right) x(t)+\sum_{i \neq j_{1}, \ldots, j_{p}} b_{i} \kappa_{i}^{T} r+\sum_{i \neq j_{1}, \ldots, j_{p}} b_{i} \theta_{i}+\sum_{j=j_{1}, \ldots, j_{p}} b_{j} \bar{u}_{j} \\
y(t) & =C x(t) .
\end{aligned}
$$

For the case of $m_{r}=1$ under consideration, we have $\kappa_{i}^{T}=\kappa_{i}$ as scalars, $i=1,2, \ldots, m$. For this system to match the desired closed-loop system $y(t)=W_{m}(s)[r](t), K, \kappa$ and $\theta$ need to be chosen to make

$$
\begin{gathered}
C\left(s I_{n}-A-\sum_{i \neq j_{1}, \ldots, j_{p}} b_{i} K_{i}^{T}\right)^{-1} \sum_{i \neq j_{1}, \ldots, j_{p}} b_{i} \kappa_{i}^{T}=W_{m}(s) \\
C\left(s I_{n}-A-\sum_{i \neq j_{1}, \ldots, j_{p}} b_{i} K_{i}^{T}\right)^{-1}\left(\sum_{i \neq j_{1}, \ldots, j_{p}} b_{i} \theta_{i}+\sum_{j=j_{1}, \ldots, j_{p}} b_{j} \bar{u}_{j}\right)=0 .
\end{gathered}
$$

The necessary and sufficient conditions for the existence of the parameters $K_{i}, \kappa_{i}$ and $\theta_{i}, i \neq j_{1}, \ldots, j_{p}$, to satisfy the above equalities were given in Ref. [49]. (The parameters depend on the failure pattern $\sigma$ and values $\left.\bar{u}_{j}\right)$. A sufficient condition for the existence of stable plant-model matching is that $\left(C, A, b_{i}\right)$ has all its zeros stable and the same relative degree as that of $W_{m}(s)=\frac{1}{P_{m}(s)}\left(\right.$ where $P_{m}(s)$ is a stable polynomial) for $i=1,2, \ldots, m$. This is also a necessary condition for the plant-model matching subject to all failure patterns resulting from up to $m-1$ actuator failures. Furthermore, since the parameters $K_{j}, \kappa_{j}$ and $\theta_{j}$, $j=j_{1}, \ldots, j_{p}$ (corresponding to failed actuators) do not have any effect on the closed-loop system, they can be arbitrarily set to zero:

$$
K_{j}=0, \kappa_{j}=0, \theta_{j}=0, j=j_{1}, \ldots, j_{p}
$$

Although the matching parameters $K, \kappa$ and $\theta$ depend on the failure conditions (patterns and values) (that is, $K=K_{\sigma}, \kappa=\kappa_{\sigma}$ and $\theta=\theta_{\sigma}$ ), they are not unique even for a given failure pattern $\sigma$, due to redundant actuation which leads to an overparametrized controller structure. For closed-loop stability, all the system pole-zero cancellations (for achieving $y(t)=W_{m}(s)[r](t)$ ) must be stable. Hence it is an important to specify the design conditions that ensure stable pole-zero cancellations. The system actuation redundancy (that is, the availability of unfailed actuators in $u=\left[u_{1}, u_{2}, \ldots, u_{m}\right]^{T}$ ), while necessary for providing failure compensation, also introduces the technical challenge of avoiding possible unstable pole-zero cancellations due to the overparametrized controller structure.

\section{IV.B. Adaptive Control with Actuation Redundancy}

The adaptive version of the nominal controller (20) has the form $(9): v(t)=\widehat{K}(t) x(t)+\widehat{\kappa}(t) r(t)+\widehat{\theta}(t)$, for which the controller parameters $\widehat{K}, \widehat{\kappa}$ and $\widehat{\theta}$ need to satisfy two key conditions:

1. it should lead to an appropriately parametrized error system in terms of the tracking error $e(t)=$ $y(t)-y_{m}(t)$ and the parameter errors $\widetilde{K}(t)=\widehat{K}(t)-K, \widetilde{\kappa}(t)=\widehat{\kappa}(t)-\kappa$ and $\widetilde{\theta}(t)=\widehat{\theta}(t)-\theta$, and

2. it should not violate the design conditions of a stable model reference adaptive control scheme, in particular, the minimum phase condition

The first condition can be satisfied with the above parameters $K=K_{\sigma}, \kappa=\kappa_{\sigma}$ and $\theta=\theta_{\sigma}$, while the second condition is not automatically satisfied even if the adaptation laws for the controller parameters $\widehat{K}, \widehat{\kappa}$ and $\widehat{\theta}$ have desirable properties, i.e., bounded parameter estimates, $L^{2}$ parameter variations, and $L^{2}$ 
estimation errors (This aspect is different from the state feedback state tracking case, where a stricter plantmodel matching condition (A.2) is needed). In the output tracking case where the plant-model matching condition is much less restrictive, some additional actuation coordination is needed in order to ensure closedloop stability. Actuation coordination may be achieved either through certain reconstruction of the controller parameters $\widehat{K}, \widehat{\kappa}$ and $\widehat{\theta}$ generated from adaptive update laws, or through some prespecified actuation scheme. Such a reconstruction of the controller parameters can be done either by using parameter projection to ensure that the estimates $\widehat{K}, \widehat{\kappa}$ and $\widehat{\theta}$ stay in certain desired regions to meet the design conditions (this has been a well-established technique), or by modifying some of the parameters to meet a certain optimization condition. This technique is currently under development, and its goal is to relax the need for knowledge of the parameter regions for controller parameter projection.

The following subsections present the idea of using a proportional actuation scheme for adaptively controlling a set of redundant actuators needed for failure compensation.

Two different situations with actuator redundancy are considered. In the first situation, all actuators have similar physical characteristics; for example, they are segments of a multiple-segment rudder or elevator of an aircraft, or nearly identical heating devices for an oven used in semiconductor manufacturing. In the second situation, the actuators are divided into several groups and the actuators within each group have similar physical characteristics; for example, a group of four engines and a group of three rudder segments in the case of a large transport aircraft.

A logical design approach for actuation in the first situation is that all actuators are assigned equal control signals, or proportional control signals, that is,

$$
\text { Equal Actuation scheme: } v_{1}(t)=v_{2}(t)=\cdots=v_{m}(t)
$$

or

$$
\text { Proportional actuation scheme: } v_{i}(t)=\alpha_{i} v_{0}(t), i=1,2, \ldots, m
$$

for some pre-chosen constants $\alpha_{i}>0, i=1,2, \ldots, m$, where $v_{0}(t) \in R$ is a scalar state feedback control input to be designed. The equal actuation scheme is appropriate when all actuators are identical, whereas the proportional actuation scheme may be more appropriate when the actuators are similar but have different levels of control authority.

A design approach for the the second situation (grouped actuators) is to assign equal or proportional control signals to all actuators within the same group. The control signals for different groups should be designed based on a multivariable control scheme that takes into account the interactions between the outputs and the inputs from different actuator groups. (For example, Refs. [7, 8, 11]).

With a chosen equal-actuation scheme (25) or proportional actuation scheme (26) for a given application, the control task is to design a single feedback control signal $v_{0}(t)$, without knowing which of the $m$ actuators $u_{i}(t), i=1,2, \ldots, m$, have failed (that is, it is not known whether $u_{i}(t)=v_{i}(t)$ or $u_{i}(t)=\bar{u}_{i}$ ), such that the plant output $y(t)$ can track a given reference model output $y_{m}(t)$ asymptotically, despite uncertainties in the plant parameters and actuator failure parameters $\bar{u}_{i}$. The reference output $y_{m}(t)$ is generated from the reference model system given in the input-output form:

$$
y_{m}(t)=W_{m}(s)[r](t), W_{m}(s)=\frac{1}{P_{m}(s)}, y_{m}(t) \in R, r(t) \in R,
$$

where $P_{m}(s)$ is a stable polynomial of degree $n^{*}$, that is, the relative degree of $W_{m}(s)$ is $n^{*}$. (The notation $y_{m}(t)=W_{m}(s)[r](t)$ is used to indicate filtering of a signal $r(t)$ by a transfer function $\left.W_{m}(s)\right)$.

\section{IV.C. Adaptive Control Design}

Recall that the SFST case needed rather restrictive matching conditions. In contrast, for output tracking, the reference model system is chosen only using the knowledge of the relative degree of the plant $(A, B, C)$. The actuator failures are assumed to be unknown. The SFOT controller structure is chosen based on the proportional actuation scheme:

$$
\begin{aligned}
v_{i}(t) & =\alpha_{i} v_{0}(t), i=1,2, \ldots, m \\
v_{0}(t) & =\widehat{K}_{0}^{T} x(t)+\widehat{\kappa}_{0} r(t)+\widehat{\theta}_{0},
\end{aligned}
$$


where $\widehat{K}_{0} \in R^{n}, \widehat{\kappa} \in R$ and $\widehat{\theta}_{0} \in R$ (for $r(t) \in R$ ) are the estimates of some nominal parameters $K_{0} \in R^{n}$, $\kappa_{0} \in R$ and $\theta_{0} \in R$ (note that the term $\widehat{\theta}_{0}$ can be expanded to handle the general failure model (3)).

Suppose there are $p$ failed actuators associated with $\sigma$ at time $t$, that is, $u_{j}=\bar{u}_{j}, j=j_{1}, j_{2}, \ldots, j_{p}$. Then, with the controller (40), the resulting closed-loop system is

$$
\begin{aligned}
\dot{x}(t)= & \left(A+\sum_{i \neq j_{1}, \ldots, j_{p}} \alpha_{i} b_{i} K_{0}^{T}\right) x(t)+\sum_{i \neq j_{1}, \ldots, j_{p}} \alpha_{i} b_{i} \kappa_{0} r(t) \\
& +\sum_{\substack{i \neq j_{1}, \ldots, j_{p} \\
\alpha_{i}}} \alpha_{i} \theta_{0}+\sum_{j=j_{1}, \ldots, j_{p}} b_{j} \bar{u}_{j}+\sum_{i \neq j_{1}, \ldots, j_{p}} \alpha_{i} b_{i}\left(\widetilde{K}_{0}^{T} x(t)+\widetilde{\kappa}_{0} r(t)+\widetilde{\theta}_{0}\right) \\
y(t)= & C x(t),
\end{aligned}
$$

where $\widetilde{K}_{0}=\widehat{K}_{0}-K_{0}, \widetilde{\kappa}_{0}=\widehat{\kappa}_{0}-\kappa_{0}$ and $\widetilde{\theta}_{0}=\widehat{\theta}_{0}-\theta_{0}$. (The subscript $\sigma$ is dropped for convenience).

For this proportional-actuation state feedback output tracking control scheme, the design conditions are

(A.3): $\left(C, A, B\left(I_{m}-\sigma\right) \alpha\right)$ with $\alpha=\left[\alpha_{1}, \alpha_{2}, \ldots, \alpha_{m}\right]^{T}$ is stabilizable and detectable, all its zeros are stable and it has the same relative degree as that of $W_{m}(s)=\frac{1}{P_{m}(s)}$, for each $\sigma \in \Sigma$; and $(C$, $\left.A, b_{j}\right)$ has the same relative degree as that of $W_{m}(s)=\frac{1}{P_{m}(s)}$, for $j=j_{1}, j_{2}, \ldots, j_{p}$ corresponding to all the failed actuators during the system operation.

To derive the dynamics of the error system, the following technical lemma is first presented:

Lemma 1 [11] If $\left(A, \bar{b}_{i}\right)$ is controllable, there exist constant $K_{0} \in R^{n}, \kappa_{0} \in R$ and $\theta_{0 j} \in R$ such that

$$
\begin{aligned}
C\left(s I_{n}-A-\bar{b}_{i} K_{0}^{T}\right)^{-1} \bar{b}_{i} \kappa_{0}=W_{m}(s) & =\frac{1}{P_{m}(s)} \\
\frac{1}{\kappa_{0}} W_{m}(s) \theta_{0 j}+C\left(s I_{n}-A-\bar{b}_{i} K_{0}^{T}\right)^{-1} \bar{b}_{j} & =0
\end{aligned}
$$

where $\bar{b}_{i}, \bar{b}_{j} \in R^{n}$ and $P_{m}(s)$ is a monic polynomial of degree $n^{*}$, if and only if the two systems $\left(C, A, \bar{b}_{i}\right)$ and $\left(C, A, \bar{b}_{j}\right)$ have the same relative degree $n^{*}$.

With $y_{m}(t)=W_{m}(s)[r](t)$, applying this lemma to the system (29) with $\bar{b}_{i}=\sum_{i \neq j_{1}, \ldots, j_{p}} \alpha_{i} b_{i}$ and $\bar{b}_{j}=b_{j}$ for $j=j_{1}, j_{2}, \ldots, j_{p}$ respectively, it can be seen that there exist $K_{0} \in R^{n}, \kappa_{0} \in R\left(\kappa_{0} C A^{n^{*}-1} \bar{b}_{i}=1\right)$ and $\theta_{0 j} \in R$ (which are piecewise constant between changes in the failure pattern $\sigma$ ) such that

$$
\begin{aligned}
y(t)= & y_{m}(t)+W_{m}(s)\left[\frac{1}{\kappa_{0}}\left(\widetilde{K}_{0}^{T} x+\widetilde{\kappa}_{0} r+\widetilde{\theta}_{0}\right)\right](t) \\
& +W_{m}(s)\left[\frac{1}{\kappa_{0}} \theta_{0}\right](t)+W_{m}(s)\left[\frac{1}{\kappa_{0}} \sum_{j=j_{1}, j_{2}, \ldots, j_{p}} \theta_{0 j} \bar{u}_{j}\right](t)+\epsilon_{0}(t)+\epsilon_{1}(t)
\end{aligned}
$$

where $\epsilon_{0}(t)$ is related to the system initial conditions and converges to zero exponentially with time $t$ because the zeros of $\left(C, A, \sum_{i \neq j_{1}, \ldots, j_{p}} \alpha_{i} b_{i}\right)$ are stable and $P_{m}(s)$ is stable. $\epsilon_{1}(t)$ is related to the transient response due to the effect of the piecewise constant parameter variations and also converges to zero exponentially with time $t$ as there are only a finite number of changes in the piecewise constant parameters $K_{0} \in R^{n}, \kappa_{0} \in R$ and $\theta_{0 j} \in R$. The effects of $\epsilon_{0}(t)$ and $\epsilon_{1}(t)$ can be ignored in the analysis as they decay to zero.

With $\theta_{0}=-\sum_{j=j_{1}, j_{2}, \ldots, j_{p}} \theta_{0 j} \bar{u}_{j}$, from (44), we have

$$
e(t)=y(t)-y_{m}(t)=W_{m}(s) \frac{1}{\kappa_{0}}\left[\widetilde{\theta}^{T} \omega\right](t),
$$

where $\widetilde{\theta}(t)=\widehat{\theta}(t)-\theta$ with $\widehat{\theta}(t)=\left[\widehat{K}_{0}^{T}, \widehat{\kappa}_{0}, \widehat{\theta}_{0}\right]^{T}$ being the estimate of $\theta=\left[K_{0}^{T}, \kappa_{0}, \theta_{0}\right]^{T}$, a and $\omega(t)=$ $\left[x^{T}(t), r(t), 1\right]^{T}$ (when the failure values $\bar{u}_{j}$ are time-varying as in (3), the term $\theta_{0}$ can be expanded, and so can its estimate $\widehat{\theta}_{0}$ with an expanded $\omega(t)$, to accomodate the effects of varying failures).

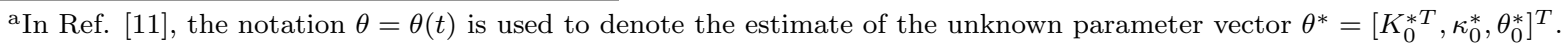


Introducing the auxiliary signals

$$
\begin{aligned}
& \zeta(t)=W_{m}(s)[\omega](t) \\
& \xi(t)=\widehat{\theta}^{T}(t) \zeta(t)-W_{m}(s)\left[\widehat{\theta}^{T} \omega\right](t) \\
& \epsilon(t)=e(t)+\widehat{\rho}(t) \xi(t),
\end{aligned}
$$

where $\widehat{\rho}(t)$ is the estimate of $\rho=\frac{1}{\kappa_{0}}=C A^{n^{*}-1} \sum_{i \neq j_{1}, \ldots, j_{p}} \alpha_{i} b_{i}$, we choose the adaptive laws as

$$
\begin{gathered}
\dot{\hat{\theta}}(t)=-\frac{\operatorname{sign}\left[\kappa_{0}\right] \Gamma \zeta(t) \epsilon(t)}{1+\zeta^{T} \zeta+\xi^{2}}, \Gamma=\Gamma^{T}>0 \\
\dot{\hat{\rho}}(t)=-\frac{\gamma \xi(t) \epsilon(t)}{1+\zeta^{T} \zeta+\xi^{2}}, \gamma>0 .
\end{gathered}
$$

$\kappa_{0}$ is a scalar gain such that $\kappa_{0} C A^{n^{*}-1} \sum_{i \neq j_{1}, \ldots, j_{p}} \alpha_{i} b_{i}=1$, so that

$$
\operatorname{sign}\left[\kappa_{0}\right]=\operatorname{sign}\left[C A^{n^{*}-1} \sum_{i \neq j_{1}, \ldots, j_{p}} \alpha_{i} b_{i}\right]=\operatorname{sign}\left[C A^{n^{*}-1} B\left(I_{m}-\sigma\right) \alpha\right]
$$

To implement this adaptive law, the following assumption is needed

(A.4): $\operatorname{sign}\left[C A^{n^{*}-1} B\left(I_{m}-\sigma\right) \alpha\right]$ does not change and is known, for each $\sigma \in \Sigma$.

This adaptive control scheme has the following stability and output tracking properties.

Theorem 2 [11] The adaptive controller (28) with the adaptive laws (37) and (38), applied to the plant (28) with actuator failures (2) guarantees that all closed-loop signals are bounded and the tracking error $e(t)=y(t)-y_{m}(t)$ goes to zero as $t \rightarrow \infty$.

This theorem can be proved using the error equation

$$
\epsilon(t)=\rho \widetilde{\theta}^{T}(t) \zeta(t)+\widetilde{\rho}(t) \xi(t)+\epsilon_{2}(t), \widetilde{\rho}=\widehat{\rho}-\rho
$$

where $\epsilon_{2}(t)$ is an exponentially decaying term, and the positive definite function

$$
V(\widetilde{\theta}, \widetilde{\rho})=|\rho| \widetilde{\theta}^{T} \Gamma^{-1} \widetilde{\theta}+\gamma^{-1} \widetilde{\rho}^{2} .
$$

From the above derivation, analysis and design, we see that the parameters $K_{0}, \kappa_{0}$ and $\theta_{0}$, which are defined through (30)-(33), are uncertain (even if the plant parameters $A, B$ and $C$ were known), due to the uncertainties in the actuator failure pattern $\sigma$ and parameters $\bar{u}_{j}$, with $j=j_{1}, j_{2}, \ldots, j_{p}$.

\section{IV.D. Discussion}

The issues with failure uncertainties and design conditions are crucial in the SFOT case. In the presence of actuator failure uncertainties, an adaptive controller is needed for $v(t)$ even if the plant parameters $A$, $B$ and $C$ were known, since the nominal controller parameters $K_{0}, \kappa_{0}$ and $\theta_{0}$ defined in (30)-(33) depend on the failure patterns and values (and even failure time instants, as $K_{0}, \kappa_{0}$ and $\theta_{0}$ are piecewise in nature and their values change when a new failure occurs). The design conditions, as stated in Assumptions (A.3) and (A.4), depend not only on the set $\Sigma$ of failure patterns but also on the actuation scheme chosen to coordinate the redundant actuators and the associated minimum phase condition for the controlled system. Such a condition is needed in order have a sufficiently large design domain required for achieving tracking of an arbitrary output trajectory.

As in the case of SFST adaptive control, the gain margin can be shown to be $(0, \infty)$ for the SFOT case under some additional conditions, ${ }^{53,54}$ i.e., it can accommodate reduced actuator effectiveness.

In view of the results obtained for SFST and SFOT, model reference adaptive control has evolved into a promising adaptive control method with systematic and rigorous design and analysis tools, and has the potential for effectively compensating for failures and damage. However, significant important open problems remain, as summarized below. 
Open problems. For direct adaptive control in the presence of actuator failures, with state feedback for output tracking, adaptive control schemes have been developed by the authors for the plant: $\dot{x}(t)=$ $A x(t)+B u(t), y(t)=C x(t)$, and the reference model system: $\dot{x}_{m}(t)=A_{m} x_{m}(t)+B_{m} r(t), y_{m}(t)=C_{m} x_{m}(t)$, with $r(t) \in R, y_{m}(t) \in R, y(t) \in R$ all being scalar signals, based on the proportional-actuation scheme: $v_{i}(t)=\alpha_{i} v_{0}(t), i=1,2, \ldots, m$, with $v_{0}(t)=\widehat{K}_{0}^{T} x(t)+\widehat{\kappa}_{0} r(t)+\widehat{\theta}_{0} \in R$. Other cases of state feedback-output tracking, with $r(t) \in R^{m_{r}}$ for $m_{r}>1$ and/or $y_{m}(t) \in R^{M}$ for $M>1$, are still open research problems. These open multi-input multi-output adaptive control problems are important for resilient aircraft flight control, because of the following reasons: (i) flight control systems can require tracking of multiple outputs, (ii) state feedback adaptive control designs are substantially simpler than output feedback control designs, (iii) the state variables are usually available for measurement, and (iv) output tracking adaptive control designs need significantly more relaxed plant-model matching conditions than state tracking designs.

Extension to nonlinear dynamic systems. An extension of the state feedback output tracking results of Section IV.C to a nonlinear system: $\dot{x}(t)=f(x(t), u(t), t), y(t)=h(x(t), t)$, was reported in Chapter 10 of Ref. [11] for the class of parametric-strict-feedback systems, with aircraft flight control applications. However, continued research is necessary for addressing more general systems.

\section{Adaptive Output Feedback Designs for Output Tracking}

This section addresses some key technical issues in direct output feedback output tracking (OFOT) adaptive control of dynamic systems in the presence of uncertain actuator failures. The issues addressed include plantmodel matching and adaptive output feedback control design and analysis for actuator failure compensation, as well as extensions to nonlinear systems.

Consider the plant: $\dot{x}(t)=A x(t)+B u(t), y(t)=C x(t)$, with $A \in R^{n \times n}, B=\left[b_{1}, b_{2}, \ldots, b_{m}\right] \in$ $R^{n \times m}, C \in R^{M \times n}$ with $M<m, x(t) \in R^{n}, y(t) \in R^{M}$, and $u(t) \in R^{m}$. The control objective is to design an adaptive output feedback control signal $v(t) \in R^{m}$ to be applied to the actuators (represented by $u(t) \in R^{m}$ ) which are subject to failures, to ensure closed-loop signal boundedness and asymptotic tracking, that is, $\lim _{t \rightarrow \infty}\left(y(t)-y_{m}(t)\right)=0$, where $y_{m}(t)$ is the output of a chosen reference model system $y_{m}(t)=W_{m}(s)[r](t) \in R^{M}$ with $r(t) \in R^{m_{r}}$ and $M \leq m_{r}$.

For output tracking, the output feedback control structure is significantly more complex than the SFOT case:

$$
v(t)=\widehat{\Theta}_{1}^{T}(t) \omega_{1}(t)+\widehat{\Theta}_{2}^{T}(t) \omega_{2}(t)+\widehat{\Theta}_{20}(t) y(t)+\widehat{\Theta}_{3}(t) r(t)+\widehat{\Theta}_{4}(t),
$$

where $\nu$ is the observability index of $(C, A)$; the controller parameters are: $\widehat{\Theta}_{1}=\left[\widehat{\Theta}_{11}, \ldots, \widehat{\Theta}_{1 \nu-1}\right]^{T}$ with $\widehat{\Theta}_{1 j} \in R^{m \times m}, j=1, \ldots, \nu-1, \widehat{\Theta}_{2}=\left[\widehat{\Theta}_{21}, \ldots, \widehat{\Theta}_{2 \nu-1}\right]^{T}$ with $\widehat{\Theta}_{2 j} \in R^{m \times M}, j=1, \ldots, \nu-1, \widehat{\Theta}_{20} \in R^{m \times M}$, $\widehat{\Theta}_{3} \in R^{m \times m_{r}}$, and $\widehat{\Theta}_{4} \in R^{m \times 1}$ for the failure model (2), (For the case of varying actuator failures (3), $\widehat{\Theta}_{4}=\widehat{\Theta}_{5}^{T}(t) \omega_{5}(t)$ for some parameter matrix $\widehat{\Theta}_{5}^{T} \in R^{m \times n_{5}}$ and unknown vector signal $\omega_{5}(t) \in R^{n_{5}}$, with some $\left.n_{5}>0\right)$, and the known signals $\omega_{1}(t)$ and $\omega_{2}(t)$ are generated from

$$
\begin{aligned}
& \omega_{1}(t)=F_{1}(s)[v](t), F_{1}(s)=\frac{A_{1}(s)}{\Lambda(s)}, A_{1}(s)=\left[I_{m}, s I_{m}, \ldots, s^{\nu-1} I_{m}\right]^{T} \\
& \omega_{2}(t)=F_{2}(s)[y](t), F_{2}(s)=\frac{A_{2}(s)}{\Lambda(s)}, A_{2}(s)=\left[I_{M}, s I_{M}, \ldots, s^{\nu-1} I_{M}\right]^{T}
\end{aligned}
$$

where $\Lambda(s)$ is a monic stable polynomial of degree $\nu-1$, and $I_{m}$ and $I_{M}$ are the $m \times m$ and $M \times M$ identity matrices respectively. Two cases of interest are: (i) $M=m_{r}=1$, and (ii) $M=m_{r}>1$, for which the above mentioned key technical issues have been addressed in Ref. [11].

\section{V.A. Plant-Model Matching}

For plant-model matching, that is, with some nominal piecewise constant parameters for the controller such that $y(t)=y_{m}(t)+\bar{\epsilon}(t)$ for some exponetially decaying $\bar{\epsilon}(t)$, the solution and design conditions can be derived for a more general case when $m \geq m_{r} \geq M \geq 1$. In this case, the nominal controller structure is

$$
v(t)=v^{*}(t)=\Theta_{1}^{T} \omega_{1}(t)+\Theta_{2}^{T} \omega_{2}(t)+\Theta_{20} y(t)+\Theta_{3} r(t)+\Theta_{4},
$$


where $\Theta_{1}, \Theta_{2}, \Theta_{20}, \Theta_{3}$ and $\Theta_{4}$ are the unknown nominal plant-model matching parameters to be estimated by $\widehat{\Theta}_{i}$ from some adaptive laws for an adaptive controller $(41) .^{\mathrm{b}}$

In the presence of actuator failures, the plant input is $u(t)=v(t)+\sigma(\bar{u}-v(t))=\left(I_{m}-\sigma\right) v(t)+\sigma \bar{u}$ as given in (4), so that the plant $\dot{x}(t)=A x(t)+B u(t), y(t)=C x(t)$, can be described as

$$
y(t)=G(s)\left[\left(I_{m}-\sigma\right) v\right](t)+G(s)[\sigma \bar{u}](t),
$$

where $G(s)=C\left(s I_{n}-A\right)^{-1} B$. Then, using (45) in (44), we have

$$
\begin{aligned}
v(t)= & \left(I_{m}-\Theta_{1}^{T} F_{1}(s)-\left(\Theta_{2}^{T} F_{2}(s)+\Theta_{20}\right) G(s)\left(I_{m}-\sigma\right)\right)^{-1} \\
& \cdot\left[\left(\Theta_{2}^{T} F_{2}(s)+\Theta_{20}\right) G(s) \sigma[\bar{u}]+\Theta_{3} r+\Theta_{4}\right](t) \\
y(t)= & G(s)\left(I_{m}-\sigma\right)\left(I_{m}-\Theta_{1}^{T} F_{1}(s)-\left(\Theta_{2}^{T} F_{2}(s)+\Theta_{20}\right) G(s)\left(I_{m}-\sigma\right)\right)^{-1} \\
& \cdot\left[\left(\Theta_{2}^{T} F_{2}(s)+\Theta_{20}\right) G(s) \sigma[\bar{u}]+\Theta_{3} r+\Theta_{4}\right](t)+G(s) \sigma[\bar{u}](t) .
\end{aligned}
$$

To make $y(t)=y_{m}(t)+\bar{\epsilon}(t)$ with $y_{m}(t)=W_{m}(s)[r](t)$ and $\lim _{t \rightarrow \infty} \bar{\epsilon}(t)=0$ exponentially, it is necessary to ensure the existence of $\Theta_{1}=\Theta_{1 \sigma}, \Theta_{2}=\Theta_{2 \sigma}, \Theta_{20}=\Theta_{20 \sigma}, \Theta_{3}=\Theta_{3 \sigma}$ and $\Theta_{4}=\Theta_{4 \sigma}$ (the subscript $\sigma$ indicates that these parameters may change as the failure pattern $\sigma$ changes) to satisfy the matching equations

$$
\begin{gathered}
G(s)\left(I_{m}-\sigma\right)\left(I_{m}-\Theta_{1}^{T} F_{1}(s)-\left(\Theta_{2}^{T} F_{2}(s)+\Theta_{20}\right) G(s)\left(I_{m}-\sigma\right)\right)^{-1} \Theta_{3}=W_{m}(s) \\
G(s)\left(I_{m}-\sigma\right)\left(I_{m}-\Theta_{1}^{T} F_{1}(s)-\left(\Theta_{2}^{T} F_{2}(s)+\Theta_{20}\right) G(s)\left(I_{m}-\sigma\right)\right)^{-1} \\
\cdot\left[\left(\Theta_{2}^{T} F_{2}(s)+\Theta_{20}\right) G(s) \sigma[\bar{u}]+\Theta_{4}\right](t)+G(s) \sigma[\bar{u}](t)=0 .
\end{gathered}
$$

for a chosen $W_{m}(s)$, for each failure pattern $\sigma \in \Sigma$, with the components $\bar{u}_{j}$ of $\bar{u}$ given in (2) or (3).

Assume that for each failure pattern $\sigma$ corresponding to a time interval $\left(T_{k}, T_{k+1}\right)$, there are $p=p(k)$ failed actuators, and that during the system operation, the maximum number of failed actuators is $p_{0}$ (that is, $p(k) \leq p_{0}$ for all $\left.k\right)$. Then, for $W_{m}(s)=P_{m}^{-1}(s)$ where $P_{m}(s)$ is an $M \times M$ polynomial matrix, and $G_{\sigma}(s)$ is the matrix containing the $m-p$ non-zero columns of $G(s)\left(I_{m}-\sigma\right)$, the above matching equations can be satisfied under the following assumption:

(A.5): for each $\sigma \in \Sigma$,

1. $\operatorname{rank}\left[G(s)\left(I_{m}-\sigma\right)\right] \geq M$, and

2. there exists an $(m-p) \times M$ constant matrix $R_{\sigma}$ such that for $G_{a}(s)=G_{\sigma}(s) R_{\sigma}$,

(a) $\operatorname{rank}\left[G_{a}(s)\right]=M$,

(b) all zeros of $G_{a}(s)$ are stable, and

(c) for $G_{a}(s)=P_{l}^{-1}(s) Z_{l a}(s)$ and $G(s) \sigma=P_{l}^{-1}(s) Z_{l b}(s)$, the transfer function matrix $Z_{l a}^{-1}(s) Z_{l b}(s)$ is proper.

For this assumption, the condition that $\operatorname{rank}\left[G(s)\left(I_{m}-\sigma\right)\right] \geq M$ implies $m-p \geq m-p_{0} \geq M$, that is, there should be at least $M$ unfailed actuators during the system operation. The conditions on $G_{a}(s)$ are standard for multivariable model reference control of $G_{\sigma}(s)$ using $v_{\sigma}(t)=R_{\sigma} z(t)$ for $z(t) \in R^{M}$ generated from a feedback control law (here, an actuator coordination condition is imposed on $R_{\sigma}$, especially because $G_{a}(s)$ is required to be minimum phase, and the condition $(2 \mathrm{c})$ that $Z_{l a}^{-1}(s) Z_{l b}(s)$ is proper means that the relative degrees of transfer functions with respect to failed actuators should be no less than those with unfailed actuators under the actuation scheme $\left.v_{\sigma}(t)=R_{\sigma} z(t)\right)$.

To establish the connection between the conditions in Assumption (A.5) and the matching equations (48) and (49), let $v_{\sigma}(t) \in R^{m-p}$ be the vector containing the components $v_{i}(t)$ of $v(t)$ corresponding to unfailed actuators such that $u_{i}(t)=v_{i}(t), i \neq j_{1}, j_{2}, \ldots, j_{p}$. In view of (44), $v_{\sigma}$ can be expressed as

$$
v_{\sigma}(t)=\bar{\Theta}_{1}^{T} \omega_{1}(t)+\bar{\Theta}_{2}^{T} \omega_{2}(t)+\bar{\Theta}_{20} y(t)+\bar{\Theta}_{3} r(t)+\bar{\Theta}_{4},
$$

\footnotetext{
${ }^{\mathrm{b}}$ In Ref. [11], $\Theta_{i}$ is used to denote the estimate of the unknown nominal parameter matrix $\Theta_{i}^{*}$, for $i=1,2,20,3,4$.
} 
where $\bar{\Theta}_{i}^{T}$ is the corresponding part of $\Theta_{i}$ in (44), for $i=1,2,20,3,4$. Then, for $R_{\sigma} \in R^{(m-p) \times M}$, we choose $\bar{\Theta}_{i}^{T}$ in (50) as $\bar{\Theta}_{i}^{T}=R_{\sigma} \Phi_{i}^{T}$ for $i=1,2,20,3,4$; in particular, for $i=1, \bar{\Theta}_{1}^{T}=R_{\sigma} \Phi_{1}^{T}$ such that $\bar{\Theta}_{1}^{T} \omega_{1}(t)=R_{\sigma} \Phi_{1}^{T} \bar{\omega}_{1}(t)$, where $\bar{\omega}_{1}(t)=\frac{\bar{A}_{1}(s)}{\Lambda(s)}[z](t)$ with $\bar{A}_{1}(s)=A_{2}(s)$ in (43) and $z(t)$ given by

$$
z(t)=\Phi_{1}^{T} \bar{\omega}_{1}(t)+\Phi_{2}^{T} \omega_{2}(t)+\Phi_{20} y(t)+\Phi_{3} r(t)+\Phi_{4} .
$$

To derive $\bar{\Theta}_{1}^{T} \omega_{1}(t)=R_{\sigma} \Phi_{1}^{T} \bar{\omega}_{1}(t)$, start by letting $\bar{\Theta}_{1}^{T} \omega_{1}(t)=R_{\sigma} \bar{\Phi}_{1}^{T} \omega_{1}(t)$ for some $\bar{\Phi}_{1}^{T}$, then set some corresponding columns of $\bar{\Phi}_{1}^{T}$ to zero to make $\bar{\Phi}_{1}^{T} \omega_{1}(t)=\overline{\bar{\Phi}}_{1}^{T} \overline{\bar{\omega}}_{1}(t)$ for $\overline{\bar{\omega}}_{1}(t)=\frac{\overline{\bar{A}}_{1}(s)}{\Lambda(s)}\left[v_{\sigma}\right](t)$ with $\overline{\bar{A}}_{1}(s)=$ $\left[I_{m-p}, s I_{m-p}, \ldots, s^{\nu-1} I_{m-p}\right]^{T}$ (recall that $v_{\sigma}(t) \in R^{m-p}$ is the non-zero part of $\left(I_{m}-\sigma\right) v(t)$ ), and finally, let $v_{\sigma}(t)=R_{\sigma} z(t)$ in $\overline{\bar{\Phi}}_{1}^{T} \frac{\bar{A}_{1}(s)}{\Lambda(s)}\left[v_{\sigma}\right](t)$ to get $\overline{\bar{\Phi}}_{1}^{T} \frac{\overline{\bar{A}}_{1}(s)}{\Lambda(s)}\left[v_{\sigma}\right](t)=\overline{\bar{\Phi}}_{1}^{T} \operatorname{diag}\left\{R_{\sigma}, \ldots, R_{\sigma}\right\} \frac{\bar{A}_{1}(s)}{\Lambda(s)}[z](t)$, that is, $\Phi_{1}^{T}=$ $\overline{\bar{\Phi}}_{1}^{T} \operatorname{diag}\left\{R_{\sigma}, \ldots, R_{\sigma}\right\}$ is the desired choice, with $\operatorname{diag}\left\{R_{\sigma}, \ldots, R_{\sigma}\right\} \in R^{(m-p)(\nu-1) \times M(\nu-1)}$.

Under the conditions on $G_{a}(s)=G_{\sigma}(s) R_{\sigma}$ in Assumption (A.5), the existence of $\Phi_{i}, i=1,2,20,3$, is guaranteed by the multivariable model reference control theory ${ }^{47}$ such that

$$
G_{a}(s)\left(I_{M}-\Phi_{1}^{T} F_{2}(s)-\left(\Phi_{2}^{T} F_{2}(s)+\Phi_{20}\right) G_{a}(s)\right)^{-1} \Phi_{3}=W_{m}(s)=P_{m}^{-1}(s),
$$

which implies that (48) can also be satisfied with some $\Theta_{1}, \Theta_{2}, \Theta_{20}, \Theta_{3}$. Moreover, under the condition that $Z_{l a}^{-1}(s) Z_{l b}(s)$ is proper, the existence of $\Phi_{4}$ is also guaranteed such that

$$
\begin{gathered}
G_{a}(s)\left(I_{M}-\Phi_{1}^{T} F_{2}(s)-\left(\Phi_{2}^{T} F_{2}(s)+\Phi_{20}\right) G_{a}(s)\right)^{-1} \\
\cdot\left[\left(\Phi_{2}^{T} F_{2}(s)+\Phi_{20}\right) G(s) \sigma[\bar{u}]+\Phi_{4}\right](t)+G(s) \sigma[\bar{u}](t)=0
\end{gathered}
$$

which leads to the existence of $\Theta_{4}$ to meet (49).

\section{V.B. Adaptive Control Design}

The existence of the controller parameters $\Theta_{1}, \Theta_{2}, \Theta_{20}, \Theta_{3}$ and $\Theta_{4}$ to meet the desired matching equations (48) and (49) is necessary for adaptive compensation of uncertain actuator failures. It is, however, not sufficient because of the actuation redundancy; that is, there are more than $M$ unfailed actuators in the system, which may not be well coordinated, leading to possible instability. To further discuss this issue, consider a plant: $\dot{x}(t)=A x(t)+B u(t), y(t)=C x(t), u(t) \in R^{m}$ and $y(t) \in R^{M}$, with $m>M$ (that is, there is actuation redundancy). Even for the no failure case, a stable adaptive control design ${ }^{50}$ uses a $^{2}$ constant matrix $R \in R^{m \times M}$ to coordinate the redundant actuators: $u(t)=R z(t)$, leading to a new plant: $y(t)=G(s) R[z](t)$, where $G(s)=C\left(s I_{n}-A\right)^{-1} B$ and $G(s) R$ is now an $M \times M$ transfer matrix which must satisfy the design conditions of model reference adaptive control (MRAC). The question is: how should such a matrix $R$ be chosen without knowing the parameters of $G(s)$ ? The open issue is: a simple adaptation of the estimate $\widehat{R}$ of $R$ may lead to a non-minimum phase plant $G(s) \widehat{R}$, so that MRAC design is not suitable for $z(t)$.

In this subsection, a proportional-actuation based adaptive control scheme is presented for a multi-input multi-output LTI plant: $\dot{x}(t)=A x(t)+B u(t), y(t)=C x(t), u(t) \in R^{m}$ and $y(t) \in R^{M}$, with $m>M>1$, subject to uncertain actuator failures. Consider the input-output form

$$
y(t)=G(s)[u](t), G(s)=C\left(s I_{n}-A\right)^{-1} B
$$

and divide the actuators into $M$ groups: $\left\{u_{11}, \ldots, u_{1 n_{1}}\right\},\left\{u_{21}, \ldots, u_{2 n_{2}}\right\}, \ldots,\left\{u_{M 1}, \ldots, u_{M n_{M}}\right\}$, with $m=$ $n_{1}+\cdots+n_{M}$. Each group has actuators of the same physical characteristics (for example, an aircraft has a group of four engines and a group of three rudder segments). Accordingly, the plant transfer matrix $G(s)$ is partitioned in the following order:

$$
G(s)=\left[G_{11}(s), \ldots, G_{1 n_{1}}(s), G_{21}(s), \ldots, G_{2 n_{2}}(s), \ldots, G_{M 1}(s), \ldots, G_{M n_{M}}(s)\right],
$$

and, for each $\left(i\right.$ th) $M$-dimensional vector transfer function $G_{i j}(s)$, the actuator $u_{i j}$ may fail.

For each group of actuators in $u_{i}(t)=\left[u_{i 1}(t), u_{i 2}(t), \ldots, u_{i n_{i}}(t)\right]^{T}, i=1,2, \ldots, M$, a proportionalactuation scheme is used:

$$
v_{i j}(t)=\alpha_{i j} v_{i 0}(t), i=1, \ldots, M, j=1,2, \ldots, n_{i},
$$


for some constants $\alpha_{i j}$ determined by the physical characteristics of the group's actuators. The control task is to design the control vector signal $v_{0}(t)=\left[v_{10}(t), \ldots, v_{M 0}(t)\right]^{T}$ such that the plant output $y(t)$ tracks a given reference output $y_{m}(t)=W_{m}(s)[r](t) \in R^{M}$ for a stable $M \times M$ transfer matrix $W_{m}(s)$ and a bounded reference input $r(t) \in R^{M}$, despite uncertain failures of actuators in each group.

For adaptive actuator failure compensation, first consider the controller structure

$$
v_{0}(t)=\widehat{\Theta}_{1}^{T}(t) \omega_{1}(t)+\widehat{\Theta}_{2}^{T}(t) \omega_{2}(t)+\widehat{\Theta}_{20}(t) y(t)+\widehat{\Theta}_{3}(t) r(t)+\widehat{\Theta}_{4}(t)
$$

where $\widehat{\Theta}_{1}(t), \widehat{\Theta}_{2}(t), \widehat{\Theta}_{20}(t), \widehat{\Theta}_{3}(t)$ and $\widehat{\Theta}_{4}(t)$ are the estimates of some nominal plant-model matching (for minimum phase systems) or pole placement (for non-minimum phase systems) parameters $\Theta_{1}, \Theta_{2}, \Theta_{20}, \Theta_{3}$ and $\Theta_{4}$. In particular, the term $\widehat{\Theta}_{4}(t)$ is for compensation of actuator failures modeled as in (2) or (3).

Suppose that at time $t$, there are $p_{i}$ failed actuators in each group, that is, $u_{i j}=\bar{u}_{i j}$ (as described in (2) or (3)), $i=1, \ldots, M, j=j_{i 1}, \ldots, j_{i p_{i}}, 0 \leq p_{i}<n_{i}$ (that is, the number of failed actuators is less than the number of actuators in each group, ensuring that there is at least one unfailed actuator in each group to provide the desired actuation function). Then the plant $y(t)=G(s)[u](t)$ can be described as

$$
y(t)=G_{a}(s)\left[v_{0}\right](t)+\bar{y}(t),
$$

where

$$
G_{a}(s)=\left[G_{a 1}(s), \ldots, G_{a M}(s)\right]=\left[\sum_{j \neq j_{11}, \ldots, j_{p_{1}}} \alpha_{1 j} G_{1 j}(s), \ldots, \sum_{j \neq j_{M 1}, \ldots, j_{M_{p_{M}}}} \alpha_{M j} G_{M j}(s)\right]
$$

is the $M \times M$ transfer matrix associated with the unfailed actuators, and

$$
\bar{y}(t)=\sum_{i=1, \ldots, M} \sum_{j=j_{i 1}, \ldots, j_{i p_{i}}} G_{i j}(s)\left[\bar{u}_{i j}\right](t)
$$

represents the effect of the actuator failures on the plant output $y(t)$.

Plant-model matching. For plant-model matching, we consider the nominal controller structure

$$
v_{0}(t)=\Theta_{1}^{T} \omega_{1}(t)+\Theta_{2}^{T} \omega_{2}(t)+\Theta_{20} y(t)+\Theta_{3} r(t)+\Theta_{4}
$$

where $\omega_{1}(t)=F(s)\left[v_{0}\right](t), \omega_{2}=F(s)[y](t), F(s)=\frac{A_{0}(s)}{\Lambda(s)}, A_{0}(s)=\left[I_{M}, s I_{M}, \ldots, s^{\nu-2} I_{M}\right]^{T}, \Lambda(s)$ is a monic stable polynomial of degree $\nu-1$, with the observability index $\nu$ of $G_{a}(s)$. The parameters $\Theta_{1}=$ $\left[\Theta_{11}, \ldots, \Theta_{1 \nu-1}\right]^{T}, \Theta_{2}=\left[\Theta_{21}, \ldots, \Theta_{2 \nu-1}\right]^{T}, \Theta_{3}, \Theta_{i j} \in R^{M \times M}, i=1,2, j=1, \ldots, \nu-1$, are for plant-model matching, and $\Theta_{4} \in R^{M}$ is used to cancel the effect of the failed actuators $\bar{u}_{i j}(t)$.

Lemma 2 Under Assumption (A.5), there exist parameters $\Theta_{1}, \Theta_{2}, \Theta_{20}, \Theta_{3}$ and $\Theta_{4}$, such that the controller (61) ensures closed-loop signal boundedness and asymptotic tracking of the reference output $y_{m}(t)$ by the plant output $y(t)$, for each failure pattern $\sigma \in \Sigma$.

Proof: The desired parameters $\Theta_{1}, \Theta_{2}, \Theta_{20}$ and $\Theta_{3}$ are solutions of $I_{M}-\Theta_{1}^{T} F(s)-\Theta_{2}^{T} F(s) G_{a}(s)-$ $\Theta_{20} G_{a}(s)=\Theta_{3} W_{m}^{-1}(s) G_{a}(s),{ }^{47}$ for $W_{m}(s)=P_{m}^{-1}(s)$ with $P_{m}(s)=\xi_{m}(s)$ being the left interactor matrix of $G_{a}(s)$. With this matching equation, using the expressions

$$
G_{a}(s)=Z_{a}(s) P^{-1}(s)=P_{l}^{-1}(s) Z_{l a}(s) ; \quad G_{i j}(s)=P_{l}^{-1}(s) Z_{i j}(s)
$$

for some $M \times M$ polynomial matrices $Z_{a}(s), P(s), P_{l}(s)$ and $Z_{l a}(s)$ and some $M$-dimensional polynomial vectors $Z_{i j}(s)$, and substituting for $G_{i j}(s)$ in Eq $(60)$ for $\bar{y}(t)$, the closed-loop system is described as

$$
\begin{aligned}
y(t) & =W_{m}(s)[r](t)+W_{m}(s) K_{p}\left[\frac{\left(-\Theta_{1}^{T} A_{0}(s) P(s)+\Lambda(s) P(s)\right) Z_{a}^{-1}(s)}{\Lambda(s)}[\bar{y}]+\Theta_{4}\right](t) \\
& \triangleq W_{m}(s)[r](t)+f_{p}(t)
\end{aligned}
$$

for some parameter matrix $K_{p}$. There also exists a polynomial matrix $P_{a}(s)^{47}$ such that

$$
\Lambda(s) I_{M}-\Theta_{1}^{T} A_{0}(s)=P_{a}(s) Z_{l a}(s), \lim _{s \rightarrow \infty} \frac{P_{a}(s) Z_{l a}(s)}{\Lambda(s)}=I_{M},
$$


so that $f_{p}(t)=W_{m}(s) K_{p}\left[\frac{P_{a}(s)}{\Lambda(s)} \sum_{i=1, \ldots, M} \sum_{j=j_{i 1}, \ldots, j_{i p_{i}}} Z_{i j}(s)\left[\bar{u}_{i j}\right]+\Theta_{4}\right](t)$. From the second equality of (63) and the condition in Assumption (A.5) that $Z_{l a}^{-1}(s) Z_{l b}(s)$ is proper (where the columns of $Z_{l b}(s)$ in $G(s) \sigma=P_{l}^{-1}(s) Z_{l b}(s)$ are $\left.Z_{i j}(s), G_{i j}(s)=P_{l}^{-1}(s) Z_{i j}(s)\right)$, it can be concluded that $\frac{P_{a}(s)}{\Lambda(s)} Z_{i j}(s)$ is proper. With this result, for $\bar{u}_{i j}(t)$ in (2) or (3), the following parametrization can be derived:

$$
\frac{P_{a}(s)}{\Lambda(s)} \sum_{i=1, \ldots, M} \sum_{j=j_{i 1}, \ldots, j_{i p_{i}}} Z_{i j}(s)\left[\bar{u}_{i j}\right](t)=-\Theta_{5}^{T} \omega_{5}(t)
$$

for some parameter matrix $\Theta_{5}$ and vector signal $\omega_{5}(t)$. With $\Theta_{4}(t)=\Theta_{5}^{T} \omega_{5}(t)$, this completes the proof. $\nabla$

Adaptive control designs. The plant parameters and actuator failures are unknown, as are the the nominal controller parameters $\Theta_{1}, \Theta_{2}, \Theta_{20}, \Theta_{3}$ and $\Theta_{4}$, which need to be adaptively estimated. Based on the above parametrization, the following adaptive controller structure is used:

$$
v_{0}(t)=\widehat{\Theta}_{1}^{T}(t) \omega_{1}(t)+\widehat{\Theta}_{2}^{T}(t) \omega_{2}(t)+\widehat{\Theta}_{20}(t) y(t)+\widehat{\Theta}_{3}(t) r(t)+\widehat{\Theta}_{5}^{T}(t) \omega_{5}(t)
$$

where $\widehat{\Theta}_{1}(t), \widehat{\Theta}_{2}(t), \widehat{\Theta}_{20}(t), \widehat{\Theta}_{3}(t)$ and $\widehat{\Theta}_{5}(t)$ are the estimates of $\Theta_{1}, \Theta_{2}, \Theta_{20}, \Theta_{3}$ and $\Theta_{5}$, respectively.

Then, the tracking error $y(t)-y_{m}(t)$ can be expressed as

$$
y(t)-y_{m}(t)=W_{m}(s)\left[\Theta_{3}^{-1} \widetilde{\Theta}^{T} \omega\right](t),
$$

for $\widetilde{\Theta}(t)=\widehat{\Theta}(t)-\Theta$, with $\Theta=\left[\Theta_{1}^{T}, \Theta_{2}^{T}, \Theta_{20}, \Theta_{3}, \Theta_{5}^{T}\right]^{T}$ and $\omega=\left[\omega_{1}^{T}, \omega_{2}^{T}, y^{T}, r^{T}, \omega_{5}^{T}\right]^{T}$.

Different schemes can be used for designing adaptive laws for updating the controller parameters (65) for model reference based adaptive actuator failure compensation designs, including one using direct estimation of $K_{p}=\Theta_{3}^{-1}$, and other schemes using an LDU, LDS or SDU decomposition of $K_{p}{ }^{51}$ to reduce the need for prior knowledge of the high frequency gain matrix $K_{p}$. (An LDU decomposition of a matrix is of the form: $A=L D U$, where $D$ is a diagonal matrix and $L$ and $U$ are unit lower and upper triangular matrices, i.e., having unity diagonal entries. In SDU and LDS decompositions, $S$ is a symmetric positive definite matrix).

To illustrate the first adaptive scheme, suppose: $d_{m}$ is the maximum degree of $W_{m}^{-1}(s), \Psi=\Theta_{3}^{-1}=K_{p}$, $f(s)$ is a stable polynomial of degree $d_{m}$, and $h(s)=\frac{1}{f(s)}$. Define the normalized estimation error as

$$
\epsilon(t)=\frac{\xi_{m}(s) h(s)\left[y-y_{m}\right](t)+\widehat{\Psi}(t) \xi(t)}{m^{2}(t)}
$$

where $\widehat{\Psi}(t)$ is the estimate of $\Psi$, and $\zeta(t)=h(s)[\omega](t), \xi(t)=\widehat{\Theta}^{T}(t) \zeta(t)-h(s)\left[v_{0}\right](t), m^{2}(t)=1+\zeta^{T}(t) \zeta(t)+$ $\xi^{T}(t) \xi(t)$. The adaptive laws updating the controller parameters are chosen as

$$
\begin{aligned}
\dot{\widehat{\Theta}}^{T}(t) & =-S_{p} \epsilon(t) \zeta^{T}(t), \\
\dot{\widehat{\Psi}}(t) & =-\Gamma \epsilon(t) \xi^{T}(t),
\end{aligned}
$$

where $\Gamma=\Gamma^{T}>0$ and $S_{p}$ being such that $\Gamma_{p}=K_{p}^{T} S_{p}^{-1}=\Gamma_{p}^{T}>0$ are adaptation gain matrices.

Stability analysis. Suppose the actuator failures occur at time instants $T_{i}, i=1, \ldots, m_{0}$, with $m_{0}<$ $N-M+1$ since at least $M$ of the $N$ actuators do not fail. Then within each time interval $\left(T_{i}, T_{i+1}\right)$, $i=0,1, \ldots, m_{0}$, with $T_{0}=0$ and $T_{m_{0}+1}=\infty$, the actuator failure pattern does not change. Consider the positive function

$$
V(\widetilde{\Theta}, \widetilde{\Psi})=\operatorname{tr}\left[\widetilde{\Theta} \Gamma_{p} \widetilde{\Theta}^{T}\right]+\operatorname{tr}\left[\widetilde{\Psi}^{T} \Gamma^{-1} \widetilde{\Psi}\right],
$$

which is not continuous because $\Theta$ is a piecewise constant parameter matrix, and so is $\Gamma_{p}$. It can be shown that $\dot{V}=-2 \epsilon^{T}(t) \epsilon(t) m^{2}(t) \leq 0, t \in\left(T_{i}, T_{i+1}\right), i=0,1, \ldots, m_{0}$, so that $V(\widetilde{\Theta}, \widetilde{\Psi}, t) \in L^{\infty}$, that is, $\widehat{\Theta}(t)$, $\widehat{\Psi}(t) \in L^{\infty}$, which in turns implies that $\epsilon(t) m(t) \in L^{2} \cap L^{\infty}$, and from (68), that $\dot{\hat{\Theta}}(t) \in L^{2} \cap L^{\infty}$ and $\dot{\hat{\Psi}}(t) \in L^{2} \cap L^{\infty}$. Based on these properties, the desired closed-loop signal boundedness and asymptotic output tracking can be established. ${ }^{11}$ 


\section{V.C. Discussion}

The controller structure and adaptive laws can be redesigned based on an LDU, LDS or SDU decomposition of $K_{p}$, or using reduced knowledge of $K_{p} .{ }^{47}$ The choice of the actuation gains $\alpha_{i j}$ in (56) needs some knowledge of the plant. Because of the system parameter uncertainty, the value of $\alpha_{i j}$ may be uncertain, and adaptation of such gain parameters may be needed. ${ }^{52}$ This is a new unresolved issue in adaptive control. The development of adaptive failure compensation schemes to relax the minimum phase condition is also an important topic for future research.

Adaptive actuator failure compensation control schemes have been developed in Ref. [11] for both the cases when $M=m_{r}=1$ (Chapter 4) and when $M=m_{r}>1$ (Chapter 5), and are also extended to a class of single output, output-feedback nonlinear systems with actuator failures (Chapter 11).

In Ref. [11], simulation results are presented to illustrate the adaptive compensation control designs (output feedback for output tracking, state feedback for output tracking, and state feedback for state tracking) for handling uncertain actuator failures. The examples included simplified linear models of Boeing 737 longitudinal dynamics (elevator/stabilizer failure); Boeing 737 lateral-directional dynamics (rudder/aileron failure); Boeing 747 lateral-directional dynamics (rudder failure); DC-8 lateral-directional dynamics (aileron failure); F-18 wing dynamics (aileron failure), as well as nonlinear models including twin Otter longitudinal dynamics (elevator failure); and hypersonic aircraft longitudinal dynamics (elevator failure). The simulation results show that satisfactory system responses are obtained and signal boundedness and asymptotic tracking are achieved. The transient tracking error after abrupt failures was reduced quickly to a small value by controller adaptation.

Similar to the SFST and SFOT cases, the gain margin of the OFOT adaptive control law can be shown to be $(0, \infty),{ }^{53,54}$ i.e., it can accommodate reduced actuator effectiveness.

An important open topic in this research direction is the development of adaptive failure compensation control for MIMO nonlinear systems (see Section VI.B for a more detailed discussion).

\section{Challenges in Applications to Aircraft Flight Control}

The objective of adaptive control is to maintain system stability and maneuverability, as well as achieve satisfactory tracking performance in spite of uncertainties, faults, failures, and damage. The direct adaptive control approach aims to directly adjust the controller parameters based on the difference between the actual and desired performance, without explicit detection or identification of the anomaly. In particular, the adaptive controller should be able to accommodate all actuator failures whose patterns $\sigma$ belong to a specified failure pattern set $\Sigma$. Direct adaptation of the controller parameters is designed to handle multiple failures without the knowledge of the system and failure parameters and patterns.

Sections II, III and IV discussed some open issues in the design and analysis of direct adaptive control systems for applications to aircraft flight control in the presence of uncertain system actuator failures. There are additional technical issues that need to be addressed in this important research area:

- Characterization and analysis of mathematical structure of aircraft dynamics in the presence of failures and damage

- Accommodation of external disturbances, actuator saturation, unmodeled dynamics, and matching condition violations

- Adaptive controller performance with pilot in the loop

- Adaptive control with simultaneous failures in actuators, sensors, and other components

- Flight dynamics characterization in abnormal flight conditions

- Adaptive control design for multivariable and nonlinear systems with failures

The key challenges associated with these issues are: comprehensive yet mathematically tractable modeling of aircraft systems with failures and damage, and effective handling of uncertain failures and damage through redundant actuation that employs all available control surfaces as well as engine thrusts.

\section{Aircraft dynamics under failures and damage}


Failures and damage in aircraft components can cause significant changes in the system's mathematical structure. For example, asymmetric structural damage would introduce changes in the center of mass location, moments and products of inertia, and asymmetric aerodynamic forces, resulting in strong new couplings and nonlinear behavior that is hard to predict. Large structural and parametric uncertainties pose new challenges for adaptive control designs for failure and damage compensation. The design of an effective compensation scheme should be based on a suitable aircraft model that adequately represents the failures and damage. Such models are different from the standard flight dynamics models used in the literature. A limited discussion of an aircraft model, that permits the use of engines as actuators, is briefly presented next.

Aircraft models with differential engine thrusts. A normal aircraft flight dynamics model used in the literature for flight control design is based on the assumption that the engine thrusts are symmetric and not adjusted independently. However, when engine thrusts are used differentially for compensation of anomalies such as asymmetric damage or rudder failures, this assumption is not valid, and a model with asymmetric actuation of independently adjustable engine thrusts should be used. Ref. [7] developed a simplified aircraft flight dynamic model that incorporates engine differentials, for the purpose of investigating integrated adaptive propulsion and surface control effectors. From Ref. [7], the force and moment equations for a twin engine aircraft are given by in (5):

$$
\begin{aligned}
& m(\dot{u}+q w-r v)=X-m g \sin \theta+\left(T_{L}+T_{R}\right) \cos \epsilon, \\
& m(\dot{v}+r u-p w)=Y+m g \cos \theta \sin \phi, \\
& m(\dot{w}+p v-q u)=Z+m g \cos \theta \cos \phi-\left(T_{L}+T_{R}\right) \sin \epsilon, \\
& I_{x} \dot{p}+I_{x z} \dot{r}+\left(I_{z}-I_{y}\right) q r+I_{x z} q p=L+l\left(T_{L}-T_{R}\right) \sin \epsilon, \\
& I_{y} \dot{q}+\left(I_{x}-I_{z}\right) p r+I_{x z}\left(r^{2}-p^{2}\right)=M, \\
& I_{z} \dot{r}+I_{x z} \dot{p}+\left(I_{y}-I_{x}\right) q p-I_{x z} q r=N+l\left(T_{L}-T_{R}\right) \cos \epsilon,
\end{aligned}
$$

where $T_{L}$ and $T_{R}$ are the left and right engine thrusts, and other variables and parameters are standard (the descriptions are omitted here). For a conventional aircraft model without engine differentials, $T_{L}=T_{R}$ is used in Refs. [55] and [56]. The presence of $T_{L}$ and $T_{R}$ captures the essence of the aircraft model with independent engine thrusts which can be employed for effective compensation of asymmetric actuation failures such as rudder failure, aileron failure or engine malfunction. A key feature of this aircraft model is the coupling of longitudinal and lateral motion equations.

To linearize this nonlinear aircraft model, the state and control vectors of the linearized model are $x=\left[\begin{array}{lllllllll}u & w & q & \theta & v & r & p & \phi & \psi\end{array}\right]^{T}, U=\left[\begin{array}{llllll}\delta_{e} & \delta_{t_{l}} & \delta_{t_{r}} & \delta_{a_{l}} & \delta_{a_{r}} & \delta_{r}\end{array}\right]^{T}$, where $\delta_{e}, \delta_{a_{l}}, \delta_{a_{r}}$ and $\delta_{r}$ are deflections of elevator, left and right ailerons, and rudder, and $\delta_{t_{l}}$ and $\delta_{t_{r}}$ are left and right engine throttle deviations. A linearized aircraft model can be derived as:

$$
\dot{x}=\left[\begin{array}{cc}
A_{4 \times 4}^{(1)} & A_{4 \times 5}^{(2)} \\
A_{5 \times 4}^{(3)} & A_{5 \times 5}^{(4)}
\end{array}\right] x+\left[\begin{array}{cc}
B_{4 \times 3}^{(1)} & B_{4 \times 3}^{(2)} \\
B_{5 \times 3}^{(3)} & B_{5 \times 3}^{(4)}
\end{array}\right] U, A^{(3)}=\left[\begin{array}{cccc}
0 & 0 & 0 & 0 \\
\bar{T}_{u} & \bar{T}_{w} & 0 & 0 \\
\bar{T}_{u}^{\prime} & \bar{T}_{w}^{\prime} & 0 & 0 \\
0 & 0 & 0 & 0 \\
0 & 0 & 0 & 0
\end{array}\right], B^{(3)}=\left[\begin{array}{ccc}
0 & 0 & 0 \\
0 & \bar{T}_{\delta_{t_{l}}}^{\prime \prime} & -\bar{T}_{\delta_{t_{r}}}^{\prime \prime} \\
0 & \bar{T}_{\delta_{t_{l}}^{\prime \prime}} & -\bar{T}_{\delta_{t_{r}}}^{\prime \prime \prime} \\
0 & 0 & 0 \\
0 & 0 & 0
\end{array}\right]
$$

where $A^{(2)}$ and $B^{(2)}$ are zero matrices, $A^{(1)}, A^{(4)}, B^{(1)}$ and $B^{(4)}$ are standard as in the literature, and $A^{(3)}$ and $B^{(3)}$ represent the effect of engine thrust differentials (if the left and right engine thrusts are equal, $A^{(3)}$ and $B^{(3)}$ become zero, as in Refs. [55] and [56]). This model, in which the two engine thrusts and two ailerons are taken into account separately, has built-in redundancy in the system to cope with some actuator failures, such as rudder failure or engine failure on one side. Most conventional aircraft models that assume equal engine thrusts and equal aileron angles do not capture the dynamic coupling due to asymmetric failures, which represents a large structural change that should be taken into account when designing a feedback control law. Uncertainties in the failure patterns, values, and time of occurrence introduce further challenges for aircraft flight control.

Refs. [7] and [8] presented the details of such modeling of aircraft dynamics, derived design conditions for adaptive failure compensation, and presented adaptive controllers for aircraft regulation and tracking control in the presence of unknown constant rudder, aileron and engine failures. 
Aircraft models with structural damage. It is important to understand the mathematical structure of damaged aircraft dynamics. To address this problem, recently in Refs. [57] and [58], models of aircraft systems in the presence of damage and failures have been developed for investigating effects of damage and simultaneous failure- and damage- tolerance. These models in [58] were derived assuming that nominal flight (with zero steady-state angular velocities) is still achievable with feedback control, after damage and failures, which suggests that the models can be linearized at zero angular velocity for the purpose of controller design. Models of such aircraft are much more complex (with many new dynamic couplings) than those without damage, with augmented expressions for $A^{(2)}, A^{(3)}, B^{(2)}$ and $B^{(3)}$; that is, more elements of these matrices become non-zero and take on unknown and uncertain values that depend on the system damage. Such models capture the structural characterizations of aircraft dynamics in the presence of the damage and failures in a mathematically tractable manner, and can be utilized for developing provably stable adaptive failure/damage compensation control design schemes as well as rigorous analyses. Some of the relevant questions that need to be answered are: What class of systems with failures and damage can be controlled by a chosen control scheme? How can the controller architecture and structure be expanded to handle larger classes of system failures and damage? To what extent is the linearizability assumption valid? Thus, modeling and structural analysis of aircraft dynamics in the presence of failures and damage is an important topic of research for resilient aircraft flight control.

Accommodation of external disturbances, actuator saturation, unmodeled dynamics, and matching condition violations.

The actuators (control surfaces and engines) have finite response times, as well as magnitude and rate limits, that should be taken into account by the adaptive control schemes. In addition, effects of external disturbances such as wind gusts, sensor noise, as well as unmodeled dynamics (e.g., aeroelastic modes), need to be considered. Another problem that needs further research (especially in the SFST adaptive control) is quantification of how much violation of the matching conditions can be tolerated.

\section{Adaptive controller performance with pilot in the loop}

The presence of a pilot in the loop, operating in conjunction with an adaptive controller, can affect the overall performance. Pilot dynamics have been typically represented as a linear time-invariant system with a time-delay. Additional human factors also need to be taken into account while designing and evaluating an adaptive control scheme.

\section{Adaptive control with simultaneous failures in actuators, sensors, and other components}

In addition to actuators, failures can also occur in other components such as sensors, processors (used in control law implementation) and other avionics components. Sensor failures may include random unknown biases or complete outage. Several methods have been proposed in the literature for identifying and isolating failed sensors. These methods will need to be developed and integrated in the adaptive control architecture. Other issues that need to be addressed involve digital implementation, i.e., effect of sampling time, finite word length, etc.

\section{Flight dynamics characterization in abnormal flight conditions}

Under some failures and damage conditions, an aircraft can enter a highly nonlinear and unsteady flight condition, such as spin and stall. It is important to develop mathematically tractable models of dynamic behavior, both before and after entering such flight conditions. The adaptive control scheme should prevent the aircraft from entering abnormal flight conditions, and should also accomplish recovery from such conditions. In addition to modeling, this will also necessitate further research in adaptive control of nonlinear systems.

\section{Adaptive control of multivariable nonlinear systems}

The development of reconfigurable adaptive control for resilient aircraft flight systems will require advances in the state-of-the-art in adaptive control of multivariable nonlinear systems. In the presence of damage and failures, or in abnormal flight conditions, aircraft dynamics are nonlinear, heavily coupled, and have multiple inputs and outputs.

There has been significant progress in adaptive control of linear multi-input multi-output (MIMO) systems. ${ }^{51,59-64}$ Adaptive control of single-input, single-output (SISO) nonlinear systems has recently seen significant advances, for some limited classes of nonlinear systems such as pure-feedback, output-feedback 
and feedback linearizable systems. ${ }^{48,65-67}$ There is an urgent need to systematically develop nonlinear adaptive techniques for multivariable nonlinear systems, especially, those of relevance to aircraft. As stated in the previous paragraph, this would require a thorough understanding of aircraft flight dynamics in nonlinear flight regimes outside the normal flight envelope, as well as development of realistic but tractable mathematical models to enable adaptive control strategies for prevention from entering an abnormal flight conditions, and for recovery from such flight conditions. Some of the ongoing research topics on nonlinear adaptive control include: Plant parametrization, state observer construction, a priori plant information specification, and adaptive controller design, etc. The main issues to be resolved for nonlinear control designs are different from those for linear systems that are based on linear controller structures, and are technically significantly more challenging.

To see some basic ideas in multivariable adaptive control, consider the multivariable LTI plant

$$
y(t)=G_{p}(s)[u](t)+d_{p}(t), G_{p}(s)=G(s)\left(I_{m}+\mu \Delta_{m}(s)\right)+\mu \Delta_{a}(s), \mu \geq 0
$$

where $G_{p}(s)$ is an $M \times m$ transfer matrix, $\mu \Delta_{m}(s), \mu \Delta_{a}(s)$ are the multiplicative and additive unmodeled dynamics, and $d_{p}(t)$ is a bounded disturbance. An important concept for a multivariable system is its modified left interactor $\xi_{m}(s)^{47}$ which has a stable inverse (needed for model reference adaptive control) such that the high frequency gain matrix $K_{p}=\lim _{s \rightarrow \infty} \xi_{m}(s) G(s)$ is nonsingular.

An MRAC scheme for the plant (73) with $\mu=0$ and $d_{p}(t)=0$, can be designed under the conditions: $G(s)$ full rank with known observability index (or its upper bound), known $\xi_{m}(s)$, stable zeros, and $K_{p} S_{p}=$ $\left(K_{p} S_{p}\right)^{T}>0$ for $S_{p}$ known. With the knowledge of stability margins (bounds) of $\Delta_{a}(s)$ and $\Delta_{m}(s)$, a robust MRAC scheme can be designed for the plant (73) with a sufficiently small $\mu \neq 0 .{ }^{47}$ Relaxing the knowledge of $G(s)$ for multivariable adaptive control is an important issue, for which MRAC schemes have been proposed for unknown $K_{p},{ }^{51}$ for unknown $\xi_{m}(s),{ }^{68}$ and for unknown $K_{p}$ and $\xi_{m}(s) .{ }^{64}$

Such fundamental technical problems in adaptive control of multivariable nonlinear systems are wide open research problems. For example, the characterization of system infinity zero structure (similar to the interactor matrix $\xi_{m}(s)$ in the linear system cases, which also defines the system relative degrees) and its use in feedback control design are some key issues to be resolved for advanced adaptive control of multivariable nonlinear systems. Furthermore, failure compensation using system actuator redundancy imposes additional challenges. As an illustration, consider the plant (73) with $y \in R^{M}$ and $u \in R^{m}$ for $m>M$ (that is, with more inputs than outputs). For $d_{p}=0$ and $\mu=0$, the available method ${ }^{50}$ to control this plant is to find a matrix $R \in R^{m \times p}$ such that $G(s) R$ is minimum phase so that an MRAC law for $u(t)=R v(t)$ can be designed for $y(t)=G(s) R[v](t)$. However, in the presence of actuator failures in $u(t)$, the specification of such a matrix $R$ needs to fit all possible failure patterns, a challenging task due to the uncertainties in $G(s)$. In Ref. 52 , it was demonstrated that such a problem is significant when using the rudder and ailerons to control an aircraft's roll and yaw motion, when the most suitable gain $R$ is unknown due to system uncertainty. Thus an important question is: how to adaptively choose such a gain matrix $R$ to fit all situations related to all possible failure patterns.

\section{Concluding Remarks}

Adaptive control technology offers a promising solution to the problem of maintaining stability and maneuverability of aircraft in the presence of anomaly. This paper presented direct model reference adaptive control schemes for systems with actuator failures, with a focus on theoretical results, including problem formulation, mathematical framework, closed-loop signal boundedness, and asymptotic tracking. Three types of adaptive schemes, having increasing degree of complexity, were presented: state tracking using state feedback, output tracking with state feedback, and output tracking with output feedback. A key feature of the direct adaptive control approach is that explicit fault detection and diagnosis, as well as controller reconfiguration, are not needed to ensure stability and tracking performance. Continued research will include application to detailed linear and nonlinear simulations of transport aircraft as well as dynamically scaled models; adaptive control in the presence of damage and failures; accommodation of unmodeled dynamics and disturbances; simultaneous actuator, sensor, processor, and avionics failures; and adaptive control theory for multi-input multi-output nonlinear systems. In addition, effects of actuator and rate saturation, relaxation of matching conditions, as well as discrete-time implementation issues, need to be fully investigated. 


\section{Acknowledgements}

This research was supported by NASA under the grant NNX08AB99A, and by NSF the under grant ECS0601475. The authors would like to thank Yu Liu and Qian Sang for their help to this paper.

\section{References}

${ }^{1}$ J. D. Boskovic, S.-H. Yu, and R. K. Mehra, "Stable adaptive fault-tolerant control of overactuated aircraft using multiple models, switching and tuning," Proceedings of the 1998 AIAA Guidance, Navigation and Control Conference, vol. 1, pp. 739-749, 1998.

${ }^{2}$ J. D. Boskovic and R. K. Mehra, "Stable multiple model adaptive flight control for accommodation of a large class of control effector failures," Proceedings of the 1999 ACC, pp. 1920-1924.

${ }^{3}$ M. Gopinathan, J. D. Boskovic, R. K. Mehra, and C. Rago, "A multiple model predictive scheme for fault-tolerant flight control design," Proceedings of the 37th IEEE Conference on Decision and Control, pp. 1376-1381, 1998.

space0.03in

${ }^{4}$ F. Ahmed-Zaid, P. Ioannou, K. Gousman, and R. Rooney, "Accommodation of failures in the F-16 aircraft using adaptive control," IEEE Control Systems Magazine, vol. 11, no. 1, pp. 73-78, 1991.

${ }^{5}$ M. Bodson and J. E. Groszkiewicz, "Multivariable adaptive algorithms for reconfigurable flight control," IEEE Trans. on Control Systems Technology, vol. 5, no. 2, pp. 217-229, 1997.

${ }^{6}$ J. D. Boskovic, S.-H. Yu, and R. K. Mehra, "A stable scheme for automatic control reconfiguration in the presence of actuator failures," Proceedings of the 1998 ACC, pp. 2455-2459.

${ }^{7}$ Y. Liu, X. D. Tang, G. Tao, and S. M. Joshi, "Adaptive failure compensation for aircraft flight control using engine differentials: regulation," Proceedings of the 2005 AIAA Infotech@Aerospace, Washington, DC, September 2005.

${ }^{8}$ Y. Liu, X. D. Tang, G. Tao and S. M. Joshi, "Adaptive failure compensation for aircraft tracking control using engine differential model," Proceedings of the 2006 American Control Conference, pp. 5984-5989, Minneapolis, MN, June 2006.

${ }^{9}$ X. D. Tang, G. Tao and S. M. Joshi, "Virtual grouping based adaptive actuator failure compensation for MIMO nonlinear systems," IEEE Transactions on Automatic Control, vol. 50, no. 11, pp. 1775-1780, 2005.

${ }^{10}$ X. D. Tang, G. Tao and S. M. Joshi, "Adaptive actuator failure compensation for nonlinear MIMO systems with an aircraft control application," Automatica, vol. 43, no. 11, pp. 1869-1883, 2007

${ }^{11}$ G. Tao, S. H. Chen, X. D. Tang and S. M. Joshi, Adaptive Control of Systems with Actuator Failures, Springer, March 2004.

${ }^{12}$ J. Chen and R. Patton, Robust Model-Based Fault Diagnosis for Dynamic Systems, Kluwer, 1998.

${ }^{13} \mathrm{P}$. M. Frank, "Fault diagnosis in dynamic systems using analytical and knowledge-based redundancy-A survey and some new results," Automatica, vol. 26, no. 3, pp. 459-474, 1990.

${ }^{14}$ J. J. Gertler, Fault Detection and Diagnosis in Engineering Systems. Marcel Dekker, New York, 1998.

${ }^{15}$ R. Iserman and P. Balle, "Trends in the application of model-based fault detection and diagnosis of technical processes," Control Engineering Practice, vol. 5, pp. 709-719, 1997.

${ }^{16} \mathrm{P}$. Kabore and H. Wang, "On the design of fault diagnosis filters and fault tolerant control", Proceedings of the American Control Conference, San Diego, CA, June 1999.

${ }^{17}$ R. Mangoubi, Robust Estimation and Failure Detection: A Concise Treatment. Springer-Verlag, 1998.

${ }^{18} \mathrm{M}$. Polycarpou and A. B. Trunov, "Learning approach to nonlinear fault diagnosis: detectability analysis," IEEE Trans. on Automatic Control, vol. 45, no. 4, pp. 806-812, 2000.

${ }^{19}$ A. T. Vemuri and M. M. Polycarpou, "Robust nonlinear fault diagnosis in input-output systems," International Journal of Control, vol. 68, no. 2, pp. 343-360, 1997.

${ }^{20} \mathrm{H}$. Wang and S. Daley, "Actuator fault diagnosis: an adaptive observer-based technique," IEEE Trans. on Automatic Control, vol. 41, no. 7, pp. 1073-1078, 1996.

${ }^{21}$ H. Wang, Z. J. Huang and S. Daley, "On the use of adaptive updating rules for actuator and sensor fault diagnosis," Automatica, vol. 33, pp. 217-225, 1997.

${ }^{22}$ A. J. Calise, S. Lee and M. Sharma, "Development of a reconfigurable flight control law for tailless aircraft," $A I A A$ Journal of Guidance, Control, and Dynamics, vol. 25, no. 5., pp. 896-902, 2001.

${ }^{23}$ B. S. Kim, A. J. Calise, "Nonlinear flight control using neural networks," AIAA Journal of Guidance, Control, and Dynamics, vol. 20, no. 1, pp. 26-33, 1997.

${ }^{24}$ J. Leitner, A. J. Calise and J. V. R. Prasad, "Analysis of adaptive neural networks for helicopter flight controls," AIAA Journal of Guidance, Control, and Dynamics, vol. 20, no. 5., pp. 972-979, September-October 1997.

${ }^{25}$ R. T. Rysdyk and A. J. Calise, "Nonlinear adaptive flight control using neural networks," IEEE Control Systems Magazine, vol. 18, no. 6, December 1998.

${ }^{26}$ K. Wise, J. S. Brinker, A. J. Calise, D. F. Enns and M. R. Elgersma, "Direct adaptive reconfigurable flight control for a tailless advanced fighter aircraft," Int. J. Robust and Nonlinear Control, vol. 9, pp. 999-1009, 1999. 
${ }^{27}$ M. Blanke, "Fault-tolerant control systems," in Advances in Control, Highlights of ECC99, pp. 171-196, Springer, edited by P. M. Frank, 1999.

${ }^{28}$ N. E. Wu, Y. Zhang, and K. Zhou, "Detection, estimation, and accommodation of loss of control effectiveness", Int. Journal of Adaptive Control and Signal Processing, vol. 14, pp. 775-795, 2000.

${ }^{29}$ G. Yen and L. Ho, "Fault tolerant control: an intelligent sliding model control strategy", Proceedings of the American Control Conference, pp. 4204-4208, Chicago, IL, June 2000.

${ }^{30}$ Y. Zhang and J. Jiang, "Design of proportional-integral reconfigurable control systems via eigenstructure assignment", Proc. of the American Control Conference, pp. 3732-3736, Chicago, IL, June 2000.

${ }^{31}$ M. A. Demetriou and M. M. Polycarpou, "Incipient fault diagnosis of dynamical systems using online approximators," IEEE Transactions on Automatic Control, vol. 43, no. 11, pp. 1612-1617, 1998.

${ }^{32}$ Y. Diao and K. Passino, "Fault tolerant stable adaptive/fuzzy/neural control for a turbine engine," IEEE TRans. on Control Systems Technology, vol. 9, no. 3, pp. 494-509, May 2001.

${ }^{33}$ R. Iserman, "On the applicability of model based fault detection for technical process," Control Engineering Practice, vol. 2, no. 3, pp. 439-450, 1997.

${ }^{34} \mathrm{Z}$. Qu, C. Ihlefeld, J. Yufang and A. Saengdeejing, "Robust control of a class of nonlinear uncertain systems-Fault tolerance against sensor failures and subsequent recovery," Proc. of the 40th IEEE CDC, Orlando, FL, 2001.

${ }^{35} \mathrm{~J}$. D. Boskovic, S. Li, and R. K. Mehra, "Intelligent control of spacecraft in the presence of actuator failures," Proceedings of the 38th IEEE Conference on Decision and Control, pp. 4472-4477, Phoenix, AZ, December 1999.

${ }^{36}$ E. G. Alcorta and P. M. Frank, "Deterministic nonlinear observer-based approaches to fault diagnosis: A survey," Control Engineering Practice, vol. 5, no. 5, pp. 663-700, 1997.

${ }^{37}$ H. Hammouri, M. Kinnaert, and E. H. El Yaagoubi, "Observer-based approach to fault detection and isolation for nonlinear systems," IEEE Transactions on Automatic Control, vol. 44, no. 10, pp. 1879-1884, 1999.

${ }^{38}$ C. A. Jacobson and C. N. Nett, "An integrated approach to controls and diagnostic using the four parameter controller," IEEE Control Systems Magazine, vol. 11, pp. 22-29, 1991.

${ }^{39}$ X. C. Lou, A. S. Willsky, and G. C. Verghese, "Optimal robust redundancy relations for failure detection in uncertain systems," Automatica, vol. 22, no. 3, pp. 333-344.

${ }^{40}$ M. A. Massoumnia, G. C, Verghese, and A. S. Willsky, "Failure detection and identification," IEEE Transactions on Automatic Control, vol. 34, no. 3, pp. 316-321, 1989.

${ }^{41}$ R. J. Patton and Kanguette, "Robust fault diagnosis using eigenstructure assignment of observers," in Fault Diagnosis in Dynamic Systems: Theory and Application, edited by R. J. Patton, P. M. Frank, and R. N. Clark, pp. 99-154, Prentice-Hall, Englewood Cliffs, NJ, 1989.

${ }^{42}$ J. M. Maciejowski, "Reconfigurable control using constrained optimization," Proceedings of ECC95, pp. 107-130, 1995.

${ }^{43}$ H. Noura, D. Sauter, F. Hamelin, and D. Theilliol, "Fault-tolerant control in dynamic systems: application to a winding machine," IEEE Control Systems Maganize, vol. 20, pp. 33-49, 2000.

${ }^{44}$ N. E. Wu and T. J. Chen, "Feedback design in control reconfigurable systems," International Journal of Robust and Nonlinear Control, vol. 6, pp. 560-570, 1996.

${ }^{45}$ N. E. Wu, K. Zhou, and G. Salomon, "Reconfigurability in linear time-invariant systems," Automatica, vol. 36, pp. 1767-1771, 2000.

${ }^{46}$ V. Patel, C. Cao, N. Hovakimyan, K. Wise, and E. Lavretsky, " $\mathcal{L}_{1}$ adaptive controller for tailless unstable aircraft", Proc. of the American Control Conference, pp. 5272-5277, New York, NY, July 2007.

${ }^{47} \mathrm{G}$. Tao, Adaptive Control Design and Analysis, John Wiley \& Sons, 2003.

${ }^{48}$ A. Isidori, Nonlinear Control Systems, 3rd ed., Springer-Verlag, Berlin, 1995.

${ }^{49} \mathrm{G}$. Tao, X. L. Ma and S. M. Joshi, "Adaptive output tracking control of systems with actuator failures," Proceedings of the 2000 American Control Conference, pp. 2654-2658, Chicago, IL, June 2000.

${ }^{50}$ Goodwin, G. C., B. C. McInnis and J. C. Wang, "Model reference adaptive control for systems having non-square transfer functions," Proceedings of the 21st IEEE Conference on Decision and Control, pp. 744-749, Orlando, FL, 1982.

${ }^{51}$ A. K. Imai, R. R. Costa, L. Hsu, G. Tao and P. V. Kokotović, "Multivariable MRAC using high frequency gain matrix factorization," IEEE Transactions on Automatic Control, vol. 49, no. 7, pp. 1152-1157, 2004.

${ }^{52}$ X. D. Tang, Y. Liu and G. Tao, "A study of adaptation of multiple actuating signals for LTI systems," Proceedings of the 2006 American Control Conference, pp. 5996-6001, Minneapolis, MN, 2006.

${ }^{53}$ Q. Sang and G. Tao, "Gain margins of model reference adaptive control systems," Proceedings of the 7th World Congress on Intelligent Control and Automation, Chongqing, China, June 2008.

${ }^{54} \mathrm{Q}$. Sang and G. Tao, "Gain margins of multivariable model reference adaptive control systems," submitted to the 47th IEEE Conference on Decision and Control, Cancun, Mexico, 2008

${ }^{55}$ A. E. Bryson, Jr., Control of Spacecraft and Aircraft, Princeton University Press, Princeton, NJ, 1994

${ }^{56}$ G. F. Franklin, J. D. Powell, and A. Emani-Naeini, Feedback Control of Dynamic Systems, 3rd ed., Addison Wesley, 1994.

${ }^{57}$ B. J. Bacon and I. M. Gregory, "General equations of motion for a damaged asymmetric aircraft," Proc. of AIAA Guidance, Navigation, and Control Conference, AIAA-2007-6306, Hilton Head, SC, 2007. 
${ }^{58}$ Y. Liu and G. Tao, "Direct adaptive actuator failure compensation techniques with applications to aircraft flight control systems," Proceedings of 2006 AIAA Guidance, Navigation and Control Conference, Paper AIAA-2006-6554, Keystone, CO, 2006.

${ }^{59} \mathrm{H}$. Elliott, W. A. Wolovich and M. Das, "Arbitrary adaptive pole placement for linear multivariable systems," IEEE Trans. on Auto. Control, vol. 29, no. 3, pp. 221-229, 1984.

${ }^{60}$ K. S. Narendra and A. M. Annaswamy, Stable Adaptive Systems, Prent ice-Hall, Englewood Cliffs, NJ, 1989.

${ }^{61}$ S. Sastry and M. Bodson, Adaptive Control: Stability, Convergence, and Robustness, Prentice-Hall, Englewood Cliffs, NJ, 1989 .

${ }^{62}$ G. Tao and P. A. Ioannou, "Robust model reference adaptive control for multivariable plants," Int. J. of Adaptive Control and Signal Processing, vol. 2, no. 3, 1988.

${ }^{63} \mathrm{G}$. Tao and P. A. Ioannou, "Stability and robustness of multivariable model reference adaptive control schemes," in Advances in Robust Control Systems Techniques and Applications, Academic Press, edited by C. T. Leondes, vol. 53, pp. 99-123, 1992.

${ }^{64}$ S. R. Weller and G. C. Goodwin, "Hysteresis switching adaptive control of linear multivariable systems," IEEE Trans. on Automatic Control, vol. AC-39, no. 7, pp. 1360-1375, 1994.

${ }^{65}$ P. V. Kokotović, Ed., Foundations of Adaptive Control, Springer-Verlag, Berlin, 1991.

${ }^{66}$ M. Krstić, I. Kanellakopoulos, P. V. Kokotović, Nonlinear and Adaptive Control Design, John Wiley \& Sons, New York, 1995.

${ }^{67}$ S. S. Sastry and A. Isidori, "Adaptive control of linearizable systems," IEEE Trans. on Automatic Control, vol. 34, no. 11, pp. 1123-1131, 1989.

${ }^{68} \mathrm{G}$. Tao, "model reference adaptive control of multivariable plants with unknown interactor matrix," Proc. of the 29th IEEE CDC, pp. 2780-2785, 1990. 